\title{
Statistical quadrature evolution by inference for multicarrier continuous-variable quantum key distribution
}

\author{
Laszlo Gyongyosi · Sandor Imre
}

Received: 18 April 2019 / Accepted: 20 August 2019 / Published online: 3 September 2019

(C) The Author(s) 2019

\begin{abstract}
We define the statistical quadrature evolution (QE) method for multicarrier continuous-variable quantum key distribution (CVQKD). A multicarrier CVQKD protocol uses Gaussian subcarrier quantum continuous variables for information transmission. The $\mathrm{QE}$ scheme utilizes the theory of mathematical statistics and statistical information processing. The QE model is based on the Gaussian quadrature inference (GQI) framework to provide a minimal error estimate of the CV state quadratures. The QE method evaluates a unique and stable estimation of the non-observable continuous input from the measurement results and through the statistical inference method yielded from the GQI framework. We prove that the QE method minimizes the overall expected error by an estimator function and provides a viable, easily implementable, and computationally efficient way to maximize the extractable information from the observed data. The QE framework can be established in an arbitrary CVQKD protocol and measurement setting and is implementable by standard low-complexity functions, which is particularly convenient for experimental CVQKD.
\end{abstract}

Keywords Quantum key distribution · Quantum continuous variables · Quantum cryptography · CVQKD

\section{Introduction}

Continuous-variable quantum key distribution (CVQKD) provides a method to realize unconditional secure communication over standard, currently established telecommunication networks by standard telecommunication devices [1,4-6,8-13,26,31,43,44,49-51,53,59,60]. In a CVQKD setting, the information is carried by a continuousvariable quantum state that is defined in the phase space via the position and momentum quadratures. In a practical CVQKD implementation [19-22,33,37-40,42,58], the CV quantum states have a Gaussian random distribution,

Laszlo Gyongyosi parts of this work were presented in $[8,10]$.

L. Gyongyosi $(\bowtie)$

School of Electronics and Computer Science, University of Southampton, Southampton SO17 1BJ, UK

e-mail: lasgy_ph@yahoo.com

L. Gyongyosi · S. Imre

Department of Networked Systems and Services, Budapest University of Technology and Economics, Budapest 1117, Hungary

L. Gyongyosi

MTA-BME Information Systems Research Group, Hungarian Academy of Sciences, Budapest 1051, Hungary 
and the quantum channel between the sender (Alice) and receiver (Bob) is also Gaussian, because the presence of an eavesdropper (Eve) adds a white Gaussian noise into the transmission [2,3,15, 16, 23, 24, 29,34-36,45-48, 52,56].

The relevant performance attributes of the CVQKD protocols, such as secret key rates and transmission distances, still require significant improvements [6,10,43,49-51,53,59,60]. For this purpose, the multicarrier CVQKD has been recently introduced through the adaptive quadrature division modulation (AMQD) scheme [8]. The multicarrier CVQKD injects several additional degrees of freedom into the transmission, which are not available for a standard, single-carrier CVQKD setting [7,8,10,12-14,17-21,25,61,62]. In particular, these extra benefits and resources allow the realization of higher secret key rates and a higher amount of tolerable losses with unconditional security $[9,10,13,18,19]$. The achievable secret key rates in a multicarrier CVQKD setting have been proven in [13]. The secret key rates of AMQD confirm the multimode bounds determined in [52] (see the results on fundamental rateloss scaling in quantum optical communications in [52]). For further information on the bounds of private quantum communications, see [48].

Here, we propose the statistical quadrature evolution (QE) method for multicarrier CVQKD. The QE extends the results of the Gaussian Quadrature Inference (GQI) [10] to evolve a minimal error estimate of the continuous input regime from noisy discrete variables and estimates. The QE method utilizes the theory of mathematical statistics and the fundamentals of statistical information processing. While the GQI operates on the measured noisy subcarrier $\mathrm{CV}$ s to estimate the continuous spectrum of the non-observable input subcarriers, the QE scheme functionally builds on the GQI output and on the measured discrete noisy subcarrier variables to evolve the continuous regime of the non-observable single-carrier inputs. The QE method achieves a theoretically minimized magnitude error to evolve the non-observable single-carrier continuous variable Gaussian quadratures from the observed discrete variables. The theoretical minimum error of QE is functionally provided by the GQI method [10] and by an optimal estimator function applied to the measurement results.

The QE block evaluates a unique estimation of the non-observable continuous input regime via the noisy subcarrier variables yielded from the measurements and by the subcarrier estimations yielded from the GQI. Precisely, the QE model utilizes a corresponding estimator function that provides an optimal least-square formulation 1 (optimal leastsquares estimator). In fact, the estimator function is a linear operator whose coefficients depend on the GQI output. The QE framework also solves the problem of the optimal least-square estimate being a non-linear function of the observed data (e.g., discrete subcarrier variables and GQI output). Particularly, it is explained by the mathematical fact that the optimal least-square estimator is a linear function of the observed data if both the non-observable input (Gaussian CV states) and the observed data (measured CV states) are jointly Gaussian [30,32], which is exactly the case in a CVQKD setting. The QE framework provides a viable, easily implementable, and computationally efficient way to maximize the extractable information from the observed data. Using the fundamental theory that stands behind the GQI and QE blocks, the proposed QE output provided by the least-square formulation is always a unique and stable solution, which minimizes the overall expected estimation error for each individual component of the input vector. Specifically, by exploiting the statistical framework of multicarrier CVQKD, we prove that QE in a multicarrier CVQKD setting achieves a vanishing error as the number of subcarriers dedicated to a given user increases. We derive the corresponding expected error and expected variance using the statistical model of multicarrier CVQKD and demonstrate the results through numerical evidence. The error and error variances and the corresponding covariances are derived via computationally efficient, easily implementable functions.

The QE framework can be established in an arbitrary CVQKD protocol, and can be applied with homodyne or heterodyne measurement settings. The methods of the framework are implementable by standard low-complexity functions, which are particularly convenient for experimental CVQKD.

The novel contributions of our manuscript are as follows.

1. We define the statistical quadrature evolution $(\mathrm{QE})$ method for multicarrier continuous-variable quantum key distribution.

2. The QE scheme utilizes the theory of mathematical statistics and statistical information processing.

3. The QE block evaluates a unique and stable estimation of the non-observable continuous input from the measurement results and through the statistical inference method yielded from the GQI framework. 
4. The QE method minimizes the overall expected error by an estimator function and provides a viable, easily implementable, and computationally efficient way to maximize the extractable information from the observed data.

5. The QE framework can be established in an arbitrary CVQKD protocol and measurement setting and is implementable by standard low-complexity functions, which is particularly convenient for experimental CVQKD.

This paper is organized as follows. In Sect. 2, preliminary findings are summarized. Section 3 discusses the QE method for multiple access multicarrier CVQKD and derives the achievable statistical secret key rates. Numerical evidence is included in Sect. 4. Finally, Sect. 5 concludes the results. Supplemental information is included in the Appendix.

\section{Preliminaries}

For the notations and basic terms of AMQD, see Sects. A.1, A.2, and $[8,10,12-14,17-21,61,62]$.

\subsection{Gaussian quadrature inference}

In this section, we summarize the basic terms of GQI; for the further details, see [10].

According to the GQI framework, the $m x_{U_{k}, i}^{\prime}, i=0, \ldots, m-1$, noisy subcarrier CVs of $U_{k}, k=0, \ldots, K-1$, yield the $E\left(U^{-1}\left(x_{U_{k}, j}\right)\right)$ estimate of $U^{-1}\left(x_{U_{k}, j}\right)$, where $x_{U_{k}, j}$ is the quadrature component of $\varphi_{U_{k}, j}, \varphi_{U_{k}, j}$ is the $j$ th input $\mathrm{CV}$ of $U_{k}, \varphi_{U_{k}, j}=x_{U_{k}, j}+\mathrm{i} p_{U_{k}, j},\left\{x_{U_{k}, j}, p_{U_{k}, j}\right\}$ are Gaussian random quadratures, as

$E\left(U^{-1}\left(x_{U_{k}, j}\right)\right)=\left|\frac{1}{-\sum_{i=0}^{m-1}\left|\mathcal{T}_{i}\left(e^{i \theta_{\varphi_{U_{k}}, j}}\right)\right|^{2} \tilde{\lambda}_{i}}\right|$,

where function $\mathcal{T}_{i}\left(\mathrm{e}^{\mathrm{i} \theta_{\varphi_{U_{k}}, j}}\right)$ evaluates $T_{U_{k}, i}\left(\mathcal{N}_{U_{k}, i}\right)$ of $\mathcal{N}_{U_{k}, i}, \mathcal{N}_{U_{k}, i}$ is the $i$ th sub-channel of $U_{k}$, while $\tilde{\lambda}_{i}$ are optimal Lagrange multipliers.

Let

$\varphi_{U_{k}, j} \in \mathcal{N}\left(0, \mathbb{E}\left\|\varphi_{U_{k}, j}\right\|^{2}=2 \sigma_{\omega_{0}}^{2}\right)$,

where $x_{U_{k}, j} \in \mathcal{N}\left(0, \sigma_{\omega_{0}}^{2}\right), p_{U_{k}, j} \in \mathcal{N}\left(0, \sigma_{\omega_{0}}^{2}\right)$ are Gaussian random quadratures, $\sigma_{\omega_{0}}^{2}$ is the single-carrier modulation variance [8], and let the $m$ subcarrier CVs of $U_{k}$ be referred via

$\vec{\phi}_{U k}=\left[\phi_{U_{k}, 0} \ldots \phi_{U_{k}, m-1}\right]^{T}$,

where

$\phi_{U_{k}, i}=x_{U_{k}, i}+\mathrm{i} p_{U_{k}, i}, \phi_{U_{k}, i} \in \mathcal{N}\left(0, \mathbb{E}\left\|\phi_{U_{k}, i}\right\|^{2}=2 \sigma_{\omega_{i}}^{2}\right)$,

while $x_{U_{k}, i} \in \mathcal{N}\left(0, \sigma_{\omega_{i}}^{2}\right), p_{U_{k}, i} \in \mathcal{N}\left(0, \sigma_{\omega_{i}}^{2}\right)$ are the subcarrier quadratures, $\sigma_{\omega_{i}}^{2}$ is the quadrature modulation variance (chosen to be constant $\sigma_{\omega_{i}}^{2}=\sigma_{\omega}^{2}$ for $\forall i$, see [8]), while $\mathcal{M}_{U_{k}}$ is the logical channel (a set of $m$ subchannels) of $U_{k}$, see (A.31).

The output of $\mathcal{N}_{U_{k}, i}$ is

$\phi_{U_{k}, i}^{\prime} \in \mathcal{N}\left(0,2\left(\sigma_{\omega_{i}}^{2}+\sigma_{\mathcal{N}_{U_{k}, i}}^{2}\right)\right)$,

where $\sigma_{\mathcal{N}_{U_{k}, i}}^{2}$ is the noise variance of $\mathcal{N}_{U_{k}, i}$, and

$\vec{\phi}_{U k}^{\prime}=\left[\phi_{U_{k}, 0}^{\prime} \ldots \phi_{U_{k}, m-1}^{\prime}\right]^{T}$, 
where $\phi_{U_{k}, i}^{\prime}=x_{U_{k}, i}^{\prime}+\mathrm{i} p_{U_{k}, i}^{\prime}$ and $x_{U_{k}, i}^{\prime}, p_{U_{k}, i}^{\prime}$ are noisy Gaussian random quadratures, $x_{U_{k}, i}^{\prime} \in \mathcal{N}\left(0, \sigma_{\omega}^{2}+\sigma_{\mathcal{N}_{i}}^{2}\right)$, $p_{U_{k}, i}^{\prime} \in \mathcal{N}\left(0, \sigma_{\omega}^{2}+\sigma_{\mathcal{N}_{i}}^{2}\right)$.

Let $n$ be the number of single carriers, $n \rightarrow \infty$, and let

$\theta_{\varphi_{U_{k}, j}}=\pi / \Omega$,

where

$\Omega=\sigma_{\omega_{0}}^{2} / \sigma_{\omega}^{2}$,

and where $\sigma_{\omega_{0}}^{2}, \sigma_{\omega}^{2}$ are the single-carrier and multicarrier modulation variances, respectively.

Statistically, in a multicarrier CVQKD setting, the following relation can be written between a single-carrier $x_{U_{k}, j}$ and subcarrier $x_{U_{k}, i}$ Gaussian quadrature component

$x_{U_{k}, i}=\sum_{j=-\infty}^{\infty} x_{U_{k}, j} \mathrm{e}^{\mathrm{i} j \theta_{\varphi_{U_{k}}, j}}$,

and

$x_{U_{k}, j}=\frac{1}{2 \pi} \int_{-\pi}^{\pi} x_{U_{k}, i} \mathrm{e}^{-\mathrm{i} t_{j} \theta_{\varphi_{U_{k}, j}}} d \theta_{\varphi_{U_{k}, j}}$.

Specifically, for any $\sigma_{\omega}^{2}<\sigma_{\omega_{0}}^{2}$, it follows that

$\Omega \neq 1$

and

$\left|\theta_{\varphi_{U_{k}, j}}\right|<\pi$

therefore, $x_{U_{k}, i}$ in (9) can be rewritten as

$x_{U_{k}, i}=\frac{1}{\Omega} \sum_{j=-\infty}^{\infty} x_{U_{k}, j} \mathrm{e}^{\mathrm{i} j \theta_{\varphi_{U_{k}}, j} \frac{1}{\Omega}}$.

Note that in (9) it is assumed that the integral of (10) exists and is invertible; thus, $x_{U_{k}, j}$ is either square integrable or absolutely integrable [30].

The $x_{U_{k}, i}^{\prime}$ noisy version of (9) is available for Bob via a corresponding $M$ measurement operator (e.g., homodyne or heterodyne measurement) performed on the noisy $\phi_{i}^{\prime} \mathrm{CV}$ state, as

$$
\begin{aligned}
x_{U_{k}, i}^{\prime} & =M\left(\phi_{i}^{\prime}\right) \\
& =M\left(\mathcal{N}_{U_{k}, i}\left(\phi_{i}\right)\right) .
\end{aligned}
$$

In particular, the $\mathcal{S}\left(\mathrm{e}^{-\mathrm{i} \theta_{\varphi_{U_{k}}, j}}\right)$ spectral density of $x_{U_{k}, j}$ can be defined via the $\left|x_{U_{k}, i}^{\prime}\right|^{2}$ expectation value of $x_{U_{k}, i}^{\prime}$, as

$\mathcal{S}\left(\mathrm{e}^{-\mathrm{i} \theta_{\varphi_{U_{k}}, j}}\right)=\mathbb{E}\left(\left|x_{U_{k}, i}^{\prime}\right|^{2}\right)$,

which is a statistical measure of the strength of the fluctuations of the subcarrier components [10,30].

It can be verified that (15) is analogous to the power spectrum $\left(\mathrm{e}^{-\mathrm{i} \theta_{\varphi_{U_{k}}, j}}\right)$ of $x_{U_{k}, j}$

$\mathcal{S}\left(\mathrm{e}^{-\mathrm{i} \theta_{\varphi_{U_{k}}, j}}\right)=\left(\mathrm{e}^{-\mathrm{i} \theta_{\varphi_{U_{k}}, j}}\right)$,

where $\left(\mathrm{e}^{-\mathrm{i} \theta_{\varphi_{U_{k}}, j}}\right) \geq 0$ is a real function of $\theta_{\varphi_{U_{k}, j}}$,

$$
\left(\mathrm{e}^{-\mathrm{i} \theta_{\varphi_{U_{k}}, j}}\right)=\sum_{g=-\infty}^{\infty} \mathcal{A}_{x_{U_{k}, j}}(g) \mathrm{e}^{-\mathrm{i} \theta_{\varphi_{U_{k}, j}} g},
$$


such that

$\left(\mathrm{e}^{-\mathrm{i} \theta_{\varphi_{U_{k}, j}}}\right)=\left(\mathrm{e}^{\mathrm{i} \theta_{\varphi_{U_{k}}, j}}\right)$,

where $\mathcal{A}_{x_{U_{k}}, j}(\cdot)$ is the autocorrelation function (autocorrelation sequence $[30,32]$ ) of $x_{U_{k}, j}$, expressed as

$\mathcal{A}_{x_{U_{k}, j}}(g)=\mathbb{E}\left(x_{U_{k}, j+g} x_{U_{k}, j}\right)$.

Without loss of generality, (15) and (17), allow us to write

$\mathcal{S}\left(\mathrm{e}^{\mathrm{i} \theta_{\varphi_{U_{k}}, j}}\right)=\mathbb{E}\left(\left|x_{U_{k}, i}^{\prime}\right|^{2}\right)$.

Using (20), the estimation of $U^{-1}\left(x_{U_{k}, j}\right)$, where $U^{-1}(\cdot)$ is the inverse CVQFT unitary operation, is expressed as

$$
\begin{aligned}
E\left(U^{-1}\left(x_{U_{k}, j}\right)\right) & =\mathcal{S}\left(e^{\mathrm{i} \theta_{\varphi_{U_{k}}, j}}\right) \\
& =\mathcal{P}\left(e^{\mathrm{i} \theta_{\varphi_{U_{k}}, j}}\right),
\end{aligned}
$$

which, by using (17), can be further evaluated as

$$
\begin{aligned}
E\left(U^{-1}\left(x_{U_{k}, j}\right)\right) & =\sum_{g=-\infty}^{\infty} \mathcal{A}_{x_{U_{k}, j}}(g) e^{-\mathrm{i} \theta_{\varphi_{U_{k}}, j} g} \\
& =\mathbb{E}\left(\left|x_{U_{k}, i}^{\prime}\right|^{2}\right) .
\end{aligned}
$$

In particular, $E\left(U^{-1}\left(x_{U_{k}, j}\right)\right)$ allows us to uniquely specify $\mathcal{A}_{x_{U_{k}, i}^{\prime}}(g)$ of a noisy subcarrier quadrature $x_{U_{k}, i}^{\prime}$ as follows.

For a noisy subcarrier quadrature $x_{U_{k}, i}^{\prime}$ of the $i$ th subcarrier $\mathrm{CV} \phi_{U_{k}, i}^{\prime}$ of $U_{k}$

$\mathcal{A}_{x_{U_{k}, i}^{\prime}}(g)=\mathcal{A}_{x_{U_{k}, j}}(\Omega g)$,

where $\Omega$ is defined in (8), while $\mathcal{A}_{x_{U_{k}, j}}(g)$ of $x_{U_{k}, j}$ is as

$$
\begin{aligned}
\mathcal{A}_{x_{U_{k}, j}}(g) & =\frac{1}{2 \pi} \int_{-\pi}^{\pi} E\left(U^{-1}\left(x_{U_{k}, j}\right)\right) \mathcal{G}_{i}\left(e^{\mathrm{i} \theta_{\varphi_{U_{k}}, j}}\right) e^{\mathrm{i} g \theta_{\varphi_{U_{k}, j}}} d \theta_{\varphi_{U_{k}}, j} \\
& =\frac{1}{2 \pi} \int_{-\pi}^{\pi} \mathcal{S}\left(e^{-\mathrm{i} \theta_{\varphi_{U_{k}}, j}}\right) \mathcal{G}_{i}\left(e^{\mathrm{i} \theta_{\varphi_{U_{k}}, j}}\right) e^{\mathrm{i} g \theta_{\varphi_{U_{k}}, j}} d \theta_{\varphi_{U_{k}, j}} \\
& =\frac{1}{2 \pi} \int_{-\pi}^{\pi} \mathcal{P}\left(e^{\mathrm{i} \theta_{\varphi_{U_{k}}, j}}\right) \mathcal{G}_{i}\left(e^{\mathrm{i} \theta_{\varphi_{U_{k}}, j}}\right) e^{\mathrm{i} g \theta_{\varphi_{U_{k}, j}}} d \theta_{\varphi_{U_{k}, j}},
\end{aligned}
$$

where $\mathcal{G}_{i}\left(\mathrm{e}^{\mathrm{i} \theta_{\varphi_{U_{k}}, j}}\right)$ is defined as

$\mathcal{G}_{i}\left(\mathrm{e}^{\mathrm{i} \theta_{\varphi_{U_{k}}, j}}\right)=\mathcal{T}_{i}\left(\mathrm{e}^{\mathrm{i} \theta_{\varphi_{U_{k}}, j}}\right) \mathcal{T}_{i}\left(\frac{1}{\mathrm{e}^{\mathrm{i} \varphi_{\varphi_{k}}, j}}\right)$,

where

$\mathcal{T}_{i}\left(\mathrm{e}^{\mathrm{i} \theta_{\varphi_{U_{k}}, j}}\right)=\left\{\begin{array}{l}T_{i}\left(\mathcal{N}_{U_{k}, i}\right), \text { if }\left|\theta_{\varphi_{U_{k}, j}}\right| \leq \frac{\pi}{\Omega}, \\ 0, \text { otherwise. }\end{array}\right.$

Note that $T_{i}\left(\mathcal{N}_{U_{k}, i}\right)$ can be determined via a pilot CV state-based channel estimation procedure (pilot: contains no valuable information); for details, see [20]. 


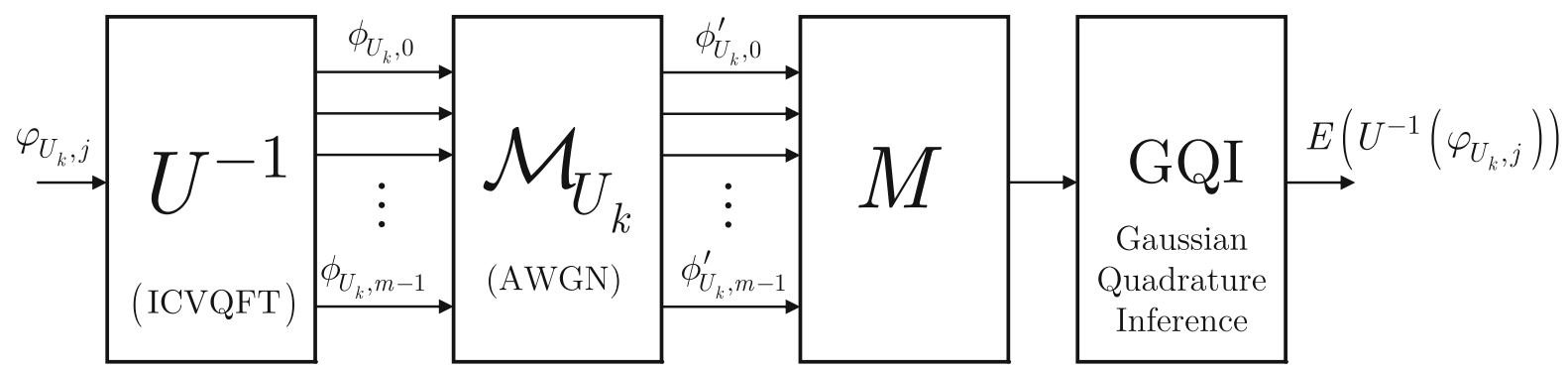

Fig. 1 The Gaussian quadrature inference (GQI) for multicarrier CVQKD [10]. User $U_{k}$ is equipped with a logical channel $\mathcal{M}_{U_{k}}=$ $\left[\mathcal{N}_{U_{k}, 0}, \ldots, \mathcal{N}_{U_{k}, m-1}\right]^{T}$, which has $m$ sub-channels. The input of $U_{k}$ is $\varphi_{U_{k}, j}=x_{U_{k}, j}+\mathrm{i} p_{U_{k}, j}$, which is transformed via the $U^{-1}$ ICVQFT operation. The $E\left(U^{-1}\left(\varphi_{U_{k}, j}\right)\right)$ estimate of the $j$ th input CV of $U_{k}, \varphi_{U_{k}, j}=x_{U_{k}, j}+\mathrm{i} p_{U_{k}, j}$ is yielded from the $m$ noisy Gaussian subcarrier CVs, $\phi_{U_{k}, i}^{\prime}=x_{U_{k}, i}^{\prime}+\mathrm{i} p_{U_{k}, i}^{\prime}, i=0, \ldots, m-1$ (ICVQFT-inverse continuous-variable quantum Fourier transform, operation $M$ refers to a heterodyne or homodyne measurement, respectively)

The $H\left(x_{U_{k}, j}\right)$ entropy rate of the Gaussian quadrature component $x_{U_{k}, j}$ of $\varphi_{U_{k}, j}$ is evaluated as

$H\left(x_{U_{k}, j}\right)=\frac{1}{2} \ln 2 \pi+\frac{1}{2}+\frac{1}{4 \pi} \int_{-\pi}^{\pi} \ln \left(\mathrm{e}^{-\mathrm{i} \theta_{\varphi_{U_{k}}, j}}\right) d \theta_{\varphi_{U_{k}, j}}$.

The GQI scheme [10] for multicarrier CVQKD is briefly summarized in Fig. 1.

Note that for a Gaussian WSS $x(n)$,

$H(x)=H\left(\mathcal{P}_{x}\right)$,

since $H(\cdot)$ is a functional of $\mathcal{P}_{x}(\cdot)[30,32]$, and

$H\left(\mathcal{S}_{x}\right)=H(x)=H\left(\mathcal{P}_{x}\right)$.

\section{Statistical quadrature evolution by Gaussian quadrature inference}

Theorem 1 (Quadrature evolution by Gaussian quadrature inference). Let $\varphi_{U_{k}, j}=x_{U_{k}, j}+\mathrm{i} p_{U_{k}, j}$ be the jth input $C V$ of user $U_{k}, k=0, \ldots, K-1$, let $E\left(U^{-1}\left(\varphi_{U_{k}, j}\right)\right)$ be the output of GQI, and let $\kappa_{U_{k}, j}=F\left(M\left(\phi_{U_{k}, i}^{\prime}\right)\right)$, $i=0, \ldots, m-1$ be the $F$-transformed $m$ noisy subcarrier $C V$ s of $U_{k}$ at a measurement $M$. The $E\left(\varphi_{U_{k}, j}\right)$ estimate of $\varphi_{U_{k}, j}$ is $E\left(\varphi_{U_{k}, j}\right)=\xi_{j} \kappa_{U_{k}, j}$, where $\xi_{j}$ is an optimal least-squares estimator yielded from $E\left(U^{-1}\left(\varphi_{U_{k}, j}\right)\right)$.

Proof Let

$\vec{\varphi}_{U_{k}}=\left(\varphi_{U_{k}, 0}, \ldots, \varphi_{U_{k}, d-1}\right)^{T}$

be a $d$-dimensional input of user $U_{k}, k=0, \ldots, K-1$, where $\varphi_{U_{k}, j}$ refers to the $j$ th single-carrier $\mathrm{CV}, 0 \leq j \leq d-1$, where $\varphi_{U_{k}, j}=x_{U_{k}, j}+\mathrm{i} p_{U_{k}, j},\left\{x_{U_{k}, j}, p_{U_{k}, j}\right\}$ are Gaussian random quadratures.

For simplicity, in later parts we refer only to the quadrature component $x_{U_{k}, j}$ of $\varphi_{U_{k}, j}$, which formulates the $d$-dimensional vector $\vec{x}_{U_{k}}$ as

$\vec{x}_{U_{k}}=\left(x_{U_{k}, 0}, \ldots, x_{U_{k}, d-1}\right)^{T}$.

Let $x_{U_{k}, i}^{\prime}$ be the $i$ th subcarrier quadrature component,

$x_{U_{k}, i}^{\prime}=M\left(\phi_{U_{k}, i}^{\prime}\right)$

resulting from a measurement $M$, where $\phi_{U_{k}, i}^{\prime}$ is the $i$ th noisy Gaussian subcarrier CV of $U_{k}$. 
Let

$\kappa_{U_{k}, j}=F\left(x_{U_{k}, i}^{\prime}\right), i=0, \ldots, m-1$,

refer to the $F$-transformed (FFT) $m$ noisy subcarrier CVs of $U_{k}$ associated with $\varphi_{U_{k}, j}$.

Applying the $F$-operation for the $x_{U_{k}, i}^{\prime}, 0 \leq i \leq m-1$, subcarriers of each $j$ of $\vec{x}_{U_{k}}$ yield the $d$-dimensional vector

$\vec{\kappa}=\left(\kappa_{U_{k}, 0}, \ldots, \kappa_{U_{k}, d-1}\right)^{T}$.

The QE block uses the GQI output $E\left(U^{-1}\left(x_{U_{k}, j}\right)\right)$, the $\kappa_{U_{k}, j}$ elements, and an optimal least-squares estimator $\xi_{j}$, evaluated via the covariances $\operatorname{cov}_{U_{U_{k, j}} \kappa_{U_{k}, j}}, \operatorname{cov}_{\kappa_{U_{k}, j} \kappa_{U_{k}, j}}$ as

$\xi_{j}=\operatorname{cov}_{x_{U_{k, j}} \kappa_{U_{k}, j}} \operatorname{cov}_{\kappa_{U_{k}, j} \kappa_{U_{k}, j}}^{-1}$,

from which $E\left(x_{U_{k}, j}\right)$ is defined as

$E\left(x_{U_{k}, j}\right)=\xi_{j} \kappa_{U_{k}, j}$.

Let $E\left(x_{U_{k}, j}\right)$ refer to the estimate of $x_{U_{k}, j}$, and let error component $\mathfrak{e}_{j}$ be defined as

$\mathfrak{e}_{j}=x_{U_{k}, j}-E\left(x_{U_{k}, j}\right)$,

with an expected error variance $E\left(\sigma_{\xi_{j}}^{2}\right)$,

$E\left(\sigma_{\xi_{j}}^{2}\right)=E\left(\mathfrak{e}_{j}^{2}\right)$

In particular, to determine the $d$-dimensional estimator $\xi$, we introduce the covariance matrices

$\operatorname{cov}_{\vec{x}_{U_{k}}} \vec{x}_{U_{k}}, \operatorname{cov}_{\vec{\kappa} \vec{\kappa}}$

and the cross-correlation [30] matrix

$\operatorname{cov}_{U_{U_{k}} \vec{\kappa}}$.

Specifically, based on a $d$-dimensional $\vec{x}_{U_{k}}$, the covariance matrix $\operatorname{cov}_{U_{U_{k}}} \vec{x}_{U_{k}}$ is a $d \times d$ dimensional matrix, evaluated as

$\left[\operatorname{cov}_{\vec{x}_{U_{k}} \vec{x}_{U_{k}}}\right]_{q r}=\hat{\mathcal{A}}_{x_{U_{k}, j}}(q-r), 1 \leq q, r \leq d$,

where $\hat{\mathcal{A}}_{x_{U_{k}}, j}(g)$ is the autocorrelation coefficient [30] associated with input $x_{U_{k}, j}$,

$\hat{\mathcal{A}}_{x_{U_{k}, j}}(g)=\frac{1}{2 \pi} \int_{-\pi}^{\pi} E\left(U^{-1}\left(x_{U_{k}, j}\right)\right) \mathrm{e}^{\mathrm{i} g \theta_{\varphi_{U_{k}}, j}} d \theta_{\varphi_{U_{k}, j}}$,

where $E\left(U^{-1}\left(x_{U_{k}, j}\right)\right)$ is the GQI output.

Based on the $d$-dimensional $\vec{\kappa}$, and a GQI output $E\left(U^{-1}\left(x_{U_{k}, j}\right)\right)$, the $\operatorname{cov}_{\vec{\kappa} \vec{\kappa}}$ covariance matrix is expressed as

$\left[\operatorname{cov}_{\vec{\kappa} \vec{\kappa}}\right]_{q t}=\mathcal{A}_{\kappa_{U_{k}, j}}(q-t), 1 \leq q, t \leq d$,

where

$\mathcal{A}_{\kappa_{U_{k}, j}}(g)=\frac{1}{2 \pi} \int_{-\pi}^{\pi} E\left(U^{-1}\left(x_{U_{k}, j}\right)\right) \mathcal{G}_{j}\left(\mathrm{e}^{\mathrm{i} \theta_{\varphi_{U_{k}}, j}}\right) \mathrm{e}^{\mathrm{i} g \theta_{\varphi_{U_{k}}, j}} d \theta_{\varphi_{U_{k}, j}}$, 
where $\mathcal{G}_{j}\left(\mathrm{e}^{\mathrm{i} \theta_{\varphi_{U_{k}}, j}}\right)$ is as

$\mathcal{G}_{j}\left(\mathrm{e}^{\mathrm{i} \theta_{\varphi_{U_{k}}, j}}\right)=\mathcal{T}_{j}\left(\mathrm{e}^{\mathrm{i} \theta_{\varphi_{U_{k}}, j}}\right) \mathcal{T}_{j}\left(\frac{1}{\mathrm{e}^{\mathrm{i} \theta \varphi_{U_{k}, j}}}\right)$,

and where

$\mathcal{T}_{j}\left(\mathrm{e}^{\mathrm{i} \theta_{\varphi_{U_{k}}, j}}\right)=\left\{\begin{array}{l}T_{j}\left(\mathcal{N}_{U_{k}, j}\right), \text { if }\left|\theta_{\varphi_{U_{k}}, j}\right| \leq \frac{\pi}{\Omega}, \\ 0, \text { otherwise }\end{array}\right.$

such that for a noisy observation $\kappa_{U_{k}, j}$,

$\left|T_{j}\left(\mathcal{N}_{U_{k}, j}\right)\right|<1$.

Without loss of generality, the cross-correlation matrix $\operatorname{cov}_{\vec{x}_{U_{k}} \vec{\kappa}}$ is evaluated as

$$
\begin{aligned}
{\left[\operatorname{cov}_{\vec{x}_{U_{k}} \vec{\kappa}}\right]_{q t} } & =\sum_{g=-\infty}^{\infty} \tau_{j}(g) \hat{\mathcal{A}}_{x_{U_{k}, j}}((q-1-\Omega(t-1))-g) \\
& =\sum_{g=-\infty}^{\infty} \tau_{j}(g) \hat{\mathcal{A}}_{x_{U_{k}, j}}((\Omega-\Omega t+q-1)-g),
\end{aligned}
$$

where $\hat{\mathcal{A}}_{x_{U_{k}, j}}(\cdot)$ is given in (42), while $\tau_{j}(\cdot)$ is determined via (23) as

$\mathcal{A}_{x_{U_{k}, j}}(g)=\left(\tau_{j}(g) * \tau_{j}(-g)\right) * \hat{\mathcal{A}}_{x_{U_{k}, j}}(g)$,

where $*$ is the linear convolution [27,30], and

$\hat{\mathcal{A}}_{x_{U_{k}, j}}(\Omega g)=\hat{\mathcal{A}}_{x_{U_{k}, i}}(g)$,

while $\hat{\mathcal{A}}_{x_{U_{k}, i}}(\cdot)$ refers to the autocorrelation coefficient associated with the $x_{U_{k}, i}$ subcarrier of user $U_{k}$.

As follows, (48) can be rewritten as

$$
\left[\operatorname{cov}_{\vec{x}_{U_{k}} \vec{\kappa}}\right]_{q t}=\sum_{g=-\infty}^{\infty} \tau_{j}(g) \frac{1}{2 \pi} \int_{-\pi}^{\pi} E\left(U^{-1}\left(x_{U_{k}, j}\right)\right) \mathrm{e}^{\mathrm{i}((\Omega-\Omega t+q-1)-g) \theta_{\varphi_{U_{k}}, j}} d \theta_{\varphi_{U_{k}, j}},
$$

by fundamental theory $[30,32]$.

Particularly, from (43) and (48), the $\xi$ optimal least-squares estimator for $d$-dimensional vectors is defined via $\operatorname{cov}_{\vec{x}_{U_{k}} \vec{\kappa}}$ and $\operatorname{cov}_{\vec{\kappa} \vec{\kappa}}$ as

$\xi=\operatorname{cov}_{\vec{x}_{U} \vec{\kappa}} \operatorname{cov}_{\vec{\kappa} \vec{\kappa}}^{-1}$,

from which $E\left(\vec{x}_{U_{k}}\right)$ is defined precisely as

$E\left(\vec{x}_{U_{k}}\right)=\xi \vec{\kappa}$.

Specifically, the estimation $E\left(\vec{x}_{U_{k}}\right)$ in (53) provides a $d$-dimensional error vector

$\mathfrak{e}=\vec{x}_{U_{k}}-E\left(\vec{x}_{U_{k}}\right)$,

which has a covariance $\operatorname{cov}_{\mathfrak{e} \mathfrak{e}}$ as

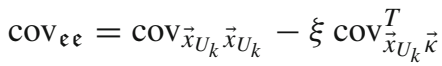

$$
\begin{aligned}
& =\operatorname{cov}_{\vec{x}_{U_{k}} \vec{x}_{U_{k}}}-\operatorname{cov}_{\vec{x}_{U_{k}} \vec{\kappa}} \operatorname{cov}_{\vec{\kappa} \vec{\kappa}}^{-1} \operatorname{cov}_{\vec{x}_{U_{k}} \vec{k}}^{T},
\end{aligned}
$$




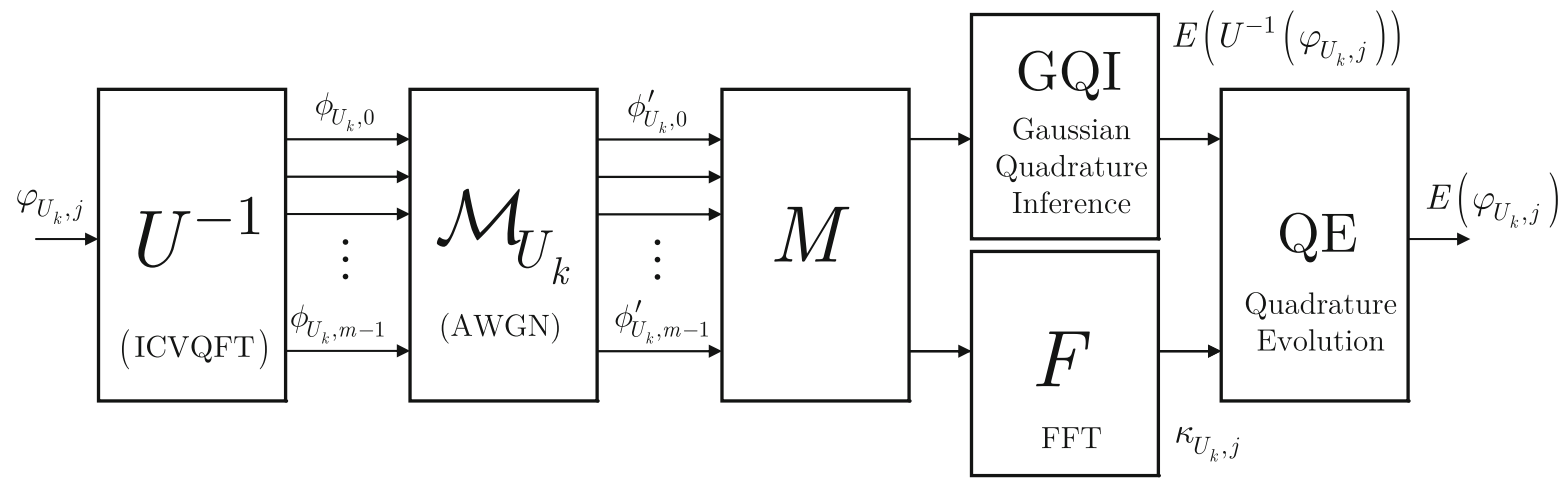

Fig. 2 Quadrature evolution (QE) by Gaussian quadrature inference (GQI). The Gaussian subcarrier CVs that are output from $\mathcal{M}_{U_{k}}$ of $U_{k}$ are measured by $M$, and fed into the GQI module. The measurement result is also transformed by the FFT (fast Fourier transform) operation. The QE module gets inputs $E\left(U^{-1}\left(\varphi_{U_{k}, j}\right)\right)$ produced by GQI and $\kappa_{U_{k}, j}=F\left(M\left(\phi_{U_{k}, i}^{\prime}\right)\right), i=0, \ldots, m-1$, and outputs the $E\left(\varphi_{U_{k}, j}\right)$ estimate of $\varphi_{U_{k}, j}$ with a theoretical error minimum

such that the overall expected estimation error, $E\{\|\mathfrak{e}\|\}$, is minimal

$\min E\left\{\|\mathfrak{e}\|^{2}\right\}=\operatorname{Tr}\left(\operatorname{cov}_{\mathfrak{e} e}\right)$,

by some fundamental theory $[30,32]$.

The $\mathcal{A}_{i}(g)$ autocorrelation coefficients are determined via the inference method of the GQE framework [10], as

$\mathcal{A}_{i}(g)=\frac{1}{2 \pi} \int_{-\pi}^{\pi}\left|\frac{\left|\mathcal{T}_{i}\left(\mathrm{e}^{\mathrm{i} \theta_{\varphi_{U}}, j}\right)\right|^{2} \mathrm{e}^{\mathrm{i} \theta_{\varphi_{U}}, j}}{\left|\mathcal{T}_{0}\left(\mathrm{e}^{\mathrm{i} \theta_{\varphi_{U}}, j}\right)\right|^{2} \lambda_{0} \cos \left(\theta_{\varphi_{U_{k}}, j}\right)}\right| d \theta_{\varphi_{U_{k}, j}}$,

where $\lambda_{0}$ is a Lagrangian coefficient.

The QE quadrature evolution scheme is depicted in Fig. 2.

\subsection{Statistical secret key rates}

Lemma 1 The $S\left(\mathcal{M}_{U_{k}}^{(m)}\right)$ statistical secret key rate converges to the $P\left(\mathcal{M}_{U_{k}}\right)$ statistical private classical information of $\mathcal{M}_{U_{k}}$, as $m \rightarrow \infty$.

Proof To derive the statistical secret key rate from the statistical quadrature evolution, we can directly apply the results to the achievable secret key rates of the GQI block from [10].

Let sub-index $(j, Z, m)$ refer to the $j$ th optimal single carrier at $Z$ autocorrelation coefficients and $m$ sub-channels in $\mathcal{M}_{U_{k}}$ dedicated to $U_{k}$. Recalling the results of Theorem 3 and Lemma 1 from [10], the following relation holds at $m$ and $m+1$ sub-channels in $\mathcal{M}_{U_{k}}$ :

$$
\begin{aligned}
D_{\mathrm{AB}}\left(\mathcal{P}_{\hat{x}_{(j ; Z, m), U_{k}}^{\prime}}\left(e^{\mathrm{i} \theta_{\varphi_{U_{k}}, j}}\right) \| \mathcal{P}_{\hat{x}_{j, U_{k}}}\left(e^{\mathrm{i} \theta_{\varphi_{U_{k}, j}}}\right)\right) & \leq D_{\mathrm{AB}}\left(\mathcal{P}_{\hat{x}_{(j ; Z, m+1), U_{k}}^{\prime}}\left(e^{\mathrm{i} \theta_{\varphi_{U_{k}}, j}}\right) \| \mathcal{P}_{\hat{x}_{j, U_{k}}}\left(e^{\mathrm{i} \theta_{\varphi_{U_{k}}, j}}\right)\right) \\
& \leq D_{\mathrm{AB}}\left(\mathcal{P}_{\hat{x}_{j, U_{k}}^{\prime}}\left(e^{\mathrm{i} \theta_{\varphi_{U_{k}, j}}}\right) \| \mathcal{P}_{\hat{x}_{j, U_{k}}}\left(e^{\mathrm{i} \theta_{\varphi_{U_{k}}, j}}\right)\right),
\end{aligned}
$$

where $D_{\mathrm{AB}}(\cdot)$ is the relative entropy between Alice and Bob, while ${\hat{\left.x^{\prime} ; Z\right), U_{k}}}^{\prime}(\cdot)$ and ${\hat{x^{\prime}}}_{(j ; Z), E}(\cdot)$ refer to the spectral densities of Bob and Eve.

Therefore, without loss of generality,

$H\left(\hat{x}_{(j ; Z, m), U_{k}}^{\prime}\left(\mathrm{e}^{\mathrm{i} \theta_{\varphi_{U_{k}}, j}}\right)\right) \geq H\left(\hat{x}_{(j ; Z, m+1), U_{k}}^{\prime}\left(\mathrm{e}^{\mathrm{i} \theta_{\varphi_{U_{k}}, j}}\right)\right) \geq H\left({\hat{x_{j}^{\prime}, U_{k}}}\left(\mathrm{e}^{\mathrm{i} \theta_{\varphi_{U_{k}}, j}}\right)\right)$. 
Let $S\left(\mathcal{M}_{U_{k}}^{(m)}\right)$ refer to the statistical secret key rate of $U_{k}$ at $m$ sub-channels in $\mathcal{M}_{U_{k}}^{(m)}$. For the GQI method, it is proven [10] that at $Z \rightarrow \infty$,

$S\left(\mathcal{M}_{U_{k}}^{(m)}\right) \leq S\left(\mathcal{M}_{U_{k}}^{(m+1)}\right)$,

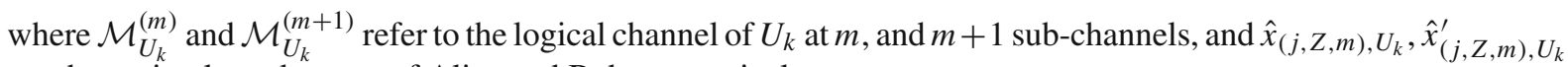
are the optimal quadratures of Alice and Bob, respectively.

Therefore, at a reverse reconciliation, for $Z \rightarrow \infty$ and $m \rightarrow \infty$

$$
\begin{aligned}
S\left(\mathcal{M}_{U_{k}}^{(m)}\right)= & \lim _{n \rightarrow \infty} \frac{1}{n} \lim _{Z \rightarrow \infty} \max _{\forall x}\left(D_{\mathrm{AB}}\left(\hat{x}_{(j ; Z), U_{k}}^{\prime} \| \hat{x}_{(j ; Z), U_{k}}\right)-D_{\mathrm{BE}}\left(\hat{x}_{(j ; Z), E}^{\prime} \| \hat{x}_{(j ; Z), U_{k}}^{\prime}\right)\right) \\
= & \lim _{n \rightarrow \infty} \frac{1}{n} \lim _{Z \rightarrow \infty} \max _{\forall x} \frac{1}{4 \pi}\left(\int_{-\pi}^{\pi}\left(\mathcal{P}_{\hat{x}_{(j ; Z), U_{k}}^{\prime}}\left(e^{\mathrm{i} \theta_{\varphi_{U_{k}}, j}}\right)-\ln \mathcal{P}_{\hat{x}_{(j ; Z), U_{k}}^{\prime}}\left(e^{\mathrm{i} \theta_{\varphi_{U_{k}, j}}}\right)-1\right) d \theta_{\varphi_{U_{k}, j}}\right. \\
& \left.-\left(\int_{-\pi}^{\pi}\left(\mathcal{P}_{\hat{x}_{(j ; Z), E}^{\prime}}\left(e^{\mathrm{i} \theta_{\varphi_{U_{k}, j}}}\right)-\ln \mathcal{P}_{\hat{x}_{(j ; Z), E}^{\prime}}\left(e^{\mathrm{i} \theta_{\varphi_{U_{k}, j}}}\right)-1\right) d \theta_{\varphi_{U_{k}, j}}\right)\right)
\end{aligned}
$$

where $D_{\mathrm{BE}}(\cdot)$ is the relative entropy between Bob and Eve.

\section{Numerical evidence}

This section proposes numerical evidence to demonstrate the results of the GQI and QE phases through a multiuser multicarrier CVQKD environment (AMQD-MQA [12]). The numerical evidence serves demonstration purposes.

\subsection{System parameters}

The numerical evidence focuses on the probability distributions of the CV states and subcarriers and studies the statistical properties and effects of modulation variance. It also revises the noise characteristic of the sub-channels and the expected errors of the quadrature estimation procedure.

The

$x_{U_{k}, j} \in \mathcal{N}\left(0, \sigma_{\omega_{0}}^{2}\right)$

single-carrier inputs of user $U_{k}$ have a modulation variance of $\sigma_{\omega_{0}}^{2}$ and formulate a $d$-dimensional input vector $\vec{x}_{U_{k}}$ (31).

The $j$ th single carrier is dedicated to a single-carrier channel $\mathcal{N}_{U_{k}, j}$. The single-carrier channel transmittance coefficient is depicted by $T\left(\mathcal{N}_{U_{k}, j}\right), 0 \leq j \leq d-1$, where $d$ is the dimension of the input vector.

The single carriers are granulated into $m$ subcarriers, where the $i$ th subcarrier is

$x_{U_{k}, i} \in \mathcal{N}\left(0, \sigma_{\omega}^{2}\right)$,

and has a modulation variance of $\sigma_{\omega}^{2}$.

The $m$ sub-channels, $\mathcal{N}_{U_{k}, i}, 0 \leq i \leq m-1$, formulate the $\mathcal{M}_{U_{k}}$ logical channel of user $U_{k}, \mathcal{M}_{U_{k}}=$ $\left[\mathcal{N}_{U_{k}, 0}, \ldots, \mathcal{N}_{U_{k}, m-1}\right]^{T}$. The $\Delta_{x_{i}} \in \mathcal{N}\left(0, \sigma_{\mathcal{N}_{U_{k}, i}}^{2}\right)$ noise variable of $\mathcal{N}_{U_{k}, i}$ is added to the subcarriers, where $\sigma_{\mathcal{N}_{U_{k}, i}}^{2}$ is the noise variance of $\mathcal{N}_{U_{k}, i}$. The transmittance coefficient of $\mathcal{N}_{U_{k}, i}$ is depicted by $T\left(\mathcal{N}_{U_{k}, i}\right)$.

The $T\left(\mathcal{N}_{U_{k}, i}\right)$ sub-channel transmittance coefficients are estimated in a pre-communication phase via the subcarrier spreading technique [20]. The subcarrier spreading is an iterative method that uses $p_{x}$ pilot CV states to statistically determine the sub-channel transmittance coefficients with minimal theoretical error. 
The outputs of the $\mathcal{N}_{U_{k}, i}$ sub-channels (noisy Gaussian subcarriers) are referred to as

$x_{U_{k}, i}^{\prime} \in \mathcal{N}\left(0, \sigma_{x_{U_{k}, i}^{\prime}}^{2}\right)$,

where

$\sigma_{x_{U_{k}, i}^{\prime}}^{2}=\sigma_{\omega}^{2}+\sigma_{\mathcal{N}_{U_{k}, i}}^{2}$.

The output of the GQI block is as

$E\left(U^{-1}\left(x_{U_{k}, j}\right)\right) \in \mathcal{N}\left(0, \tilde{\sigma}_{x_{U_{k}, i}^{\prime}}^{2}\right)$,

which at a direct-GQI (DGQI [10]) can be rewritten as

$E\left(U^{-1}\left(x_{U_{k}, j}\right)\right)=F^{-1}\left(x_{U_{k}, j}^{\prime}\right) * F^{-1}\left(\beta_{i, \varepsilon}\right)$,

where $*$ is the linear convolution, while function $\beta_{i, \varepsilon}$ provides an $\varepsilon$ minimal magnitude error,

$\varepsilon=\arg \min \varepsilon_{\max }$,

where

$\varepsilon_{\max }=\left.\max _{\forall x}|| x_{U_{k}, j}\right|^{2}-\left|x_{U_{k}, j}^{\prime}\right|^{2} \mid$,

and $x_{U_{k}, j}, x_{U_{k}, j}^{\prime}$ are the input-output single-carrier quadratures, $F^{-1}(\cdot)$ is the inverse FFT operation, while

$\beta_{i, \varepsilon}=1+\sum_{y=1}^{P} C_{y} \cos \left(y Q_{i}\right)$,

where $C_{0}$ is arbitrarily set to unity [30] and $P$ is the number of $C_{y}$ coefficients, while

$Q_{i}=\frac{2 \pi i}{m}, i=0, \ldots, m-1$.

As follows, using (67), $\tilde{\sigma}_{x_{U_{k}, i}^{\prime}}^{2}$ in (66) is evaluated as

$$
\begin{aligned}
\tilde{\sigma}_{x_{U_{k}, i}^{\prime}}^{2} & =\left(F^{-1}\left(\beta_{i, \varepsilon}\right)\right)^{2} \sigma_{x_{U_{k}, i}^{\prime}}^{2} \\
& =\left(F^{-1}\left(\beta_{i, \varepsilon}\right)\right)^{2}\left(\sigma_{\omega}^{2}+\sigma_{\mathcal{N}_{U_{k}, i}}^{2}\right),
\end{aligned}
$$

where

$F^{-1}\left(\beta_{i, \varepsilon}\right)=\sum_{i=0}^{m-1} \beta_{i, \varepsilon} \mathrm{e}^{\frac{\mathrm{i} 2 \pi i j}{m}}, j=0, \ldots, n-1$.

The $F$-transformed subcarriers formulate a $d$-dimensional vector $\vec{\kappa}(34)$, with $j$ th element as

$\kappa_{U_{k}, j} \in \mathcal{N}\left(0, \sigma_{\kappa_{U_{k}, j}}^{2}\right)$,

and with variance

$\sigma_{\kappa_{U_{k}, j}}^{2}=\sigma_{\omega_{0}}^{2}+\sigma_{\mathcal{N}_{U_{k}, j}}^{2}$.

The output of the $\mathrm{QE}$ block is a $d$-dimensional vector, with $j$ th element

$E\left(x_{U_{k}, j}\right) \in \mathcal{N}\left(0, \tilde{\sigma}_{\omega_{0}}^{2}\right)$,

with variance

$\tilde{\sigma}_{\omega_{0}}^{2}=\beta_{i, \varepsilon}^{2}\left(\sigma_{\omega_{0}}^{2}+\sigma_{\mathcal{N}_{U_{k}, j}}^{2}\right)$,

where $\beta_{i, \varepsilon}$ is given in (70).

The $j$ th single carrier is estimated from $E\left(U^{-1}\left(x_{U_{k}, j}\right)\right)$ and $\kappa_{U_{k}, j}$ as given in (36).

The $\mathfrak{e}$ estimation error is a $d$-dimensional vector with $j$ th element $\mathfrak{e}_{j}$, see (37). The $E\left(\sigma_{\xi_{j}}^{2}\right)$ expected error variance is evaluated via (38). 


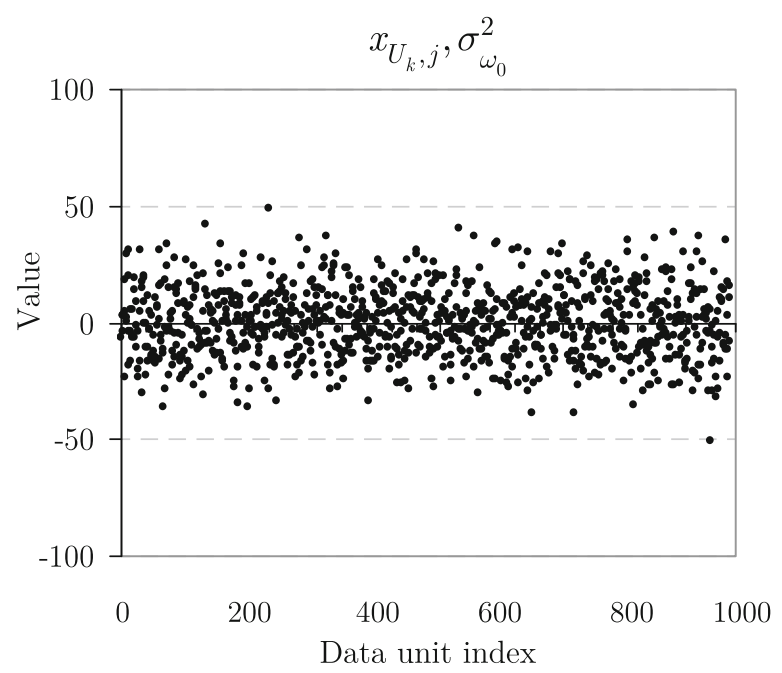

(a)

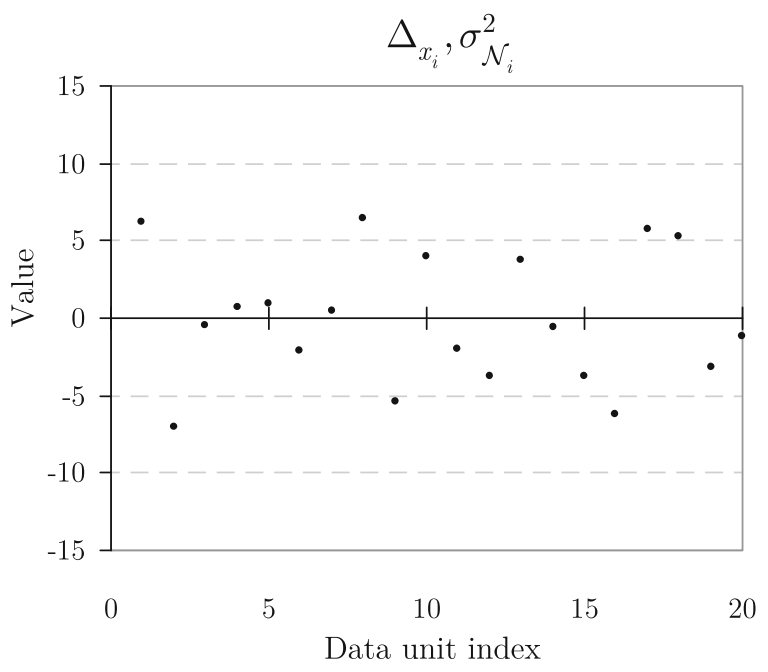

(b)

Fig. 3 a Single-carrier inputs, $x_{U_{k}, j} \in \mathcal{N}\left(0, \sigma_{\omega_{0}}^{2}\right), \sigma_{\omega_{0}}^{2}=225,0 \leq j \leq d-1, d=1000$. b The $\Delta_{x_{i}} \in \mathcal{N}\left(0, \sigma_{\mathcal{N}_{U_{k}, i}}^{2}\right)$ noise of the $m$ sub-channels of $U_{k}, \sigma_{\mathcal{N}_{U_{k}, i}}^{2}=16,0 \leq i \leq m-1, m=20$

Fig. 4 Noisy Gaussian subcarriers,

$x_{U_{k}, i}^{\prime} \in \mathcal{N}\left(0, \sigma_{x_{U_{k}, i}^{\prime}}^{2}\right)$,
$\sigma_{x_{U_{k}, i}^{\prime}}^{2}=\sigma_{\omega}^{2}+\sigma_{\mathcal{N}_{U_{k}, i}}^{2}$,
$\sigma_{\omega}^{2}=64, \sigma_{\mathcal{N}_{U_{k}, i}}^{2}=16$,
$0 \leq i \leq m-1, m=20$

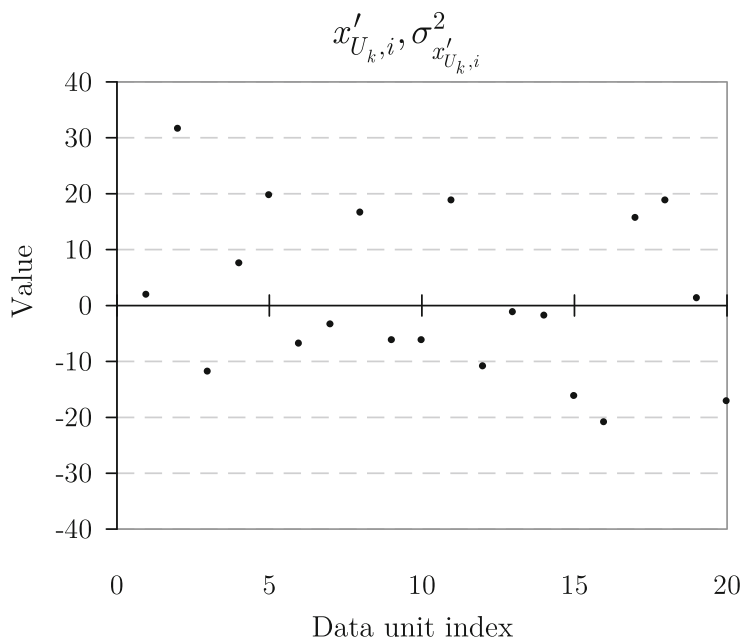

\subsection{Modulation and noise}

This analysis focuses on the probability distributions, the statistical properties of the input CV states and subcarriers, and the noise characteristics.

In Fig. 3a, the Gaussian single-carrier inputs $x_{U_{k}, j} \in \mathcal{N}\left(0, \sigma_{\omega_{0}}^{2}\right), \sigma_{\omega_{0}}^{2}=225$, of user $U_{k}$ for a $d=1000$ dimensional input vector $\vec{x}_{U_{k}}$ are depicted. In Fig. 3b, the quadrature component noise $\Delta_{x_{i}} \in \mathcal{N}\left(0, \sigma_{\mathcal{N}_{U_{k}, i}}^{2}\right)$ of the $i$ th Gaussian sub-channel $\mathcal{N}_{U_{k}, i}$ formulating the logical channel $\mathcal{M}_{U_{k}}$ of $U_{k}$ is depicted, $m=20, \sigma_{\mathcal{N}_{U_{k}, i}}^{2}=16$. The data unit index refers to single-carrier and subcarrier CV units, respectively.

In Fig. 4, the $x_{U_{k}, i}^{\prime} \in \mathcal{N}\left(0, \sigma_{x_{U_{k}, i}^{\prime}}^{2}\right), \sigma_{x_{U_{k}, i}^{\prime}}^{2}=\sigma_{\omega}^{2}+\sigma_{\mathcal{N}_{U_{k}, i}}^{2}$, outputs of the $\mathcal{N}_{U_{k}, i}$ sub-channels are depicted, $\sigma_{\omega}^{2}=64, \sigma_{\mathcal{N}_{U_{k}, i}}^{2}=16, m=20$. 


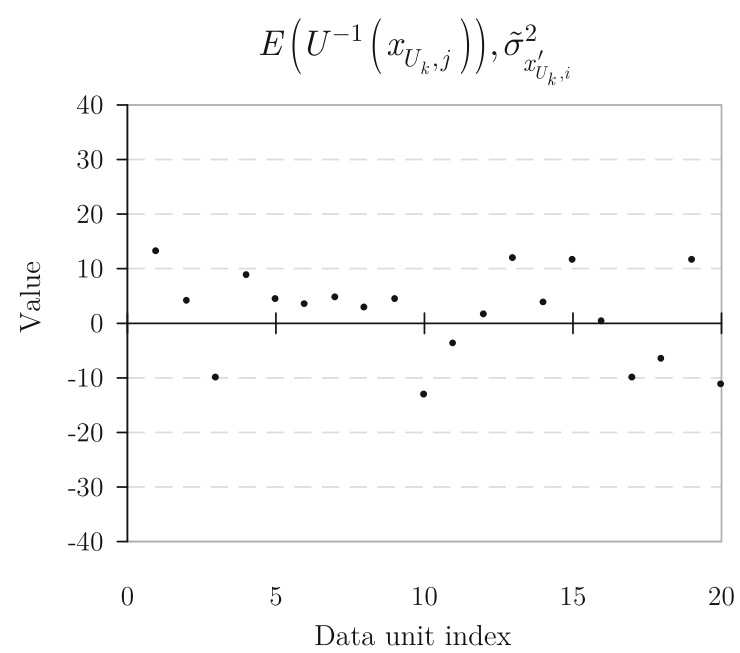

(a)

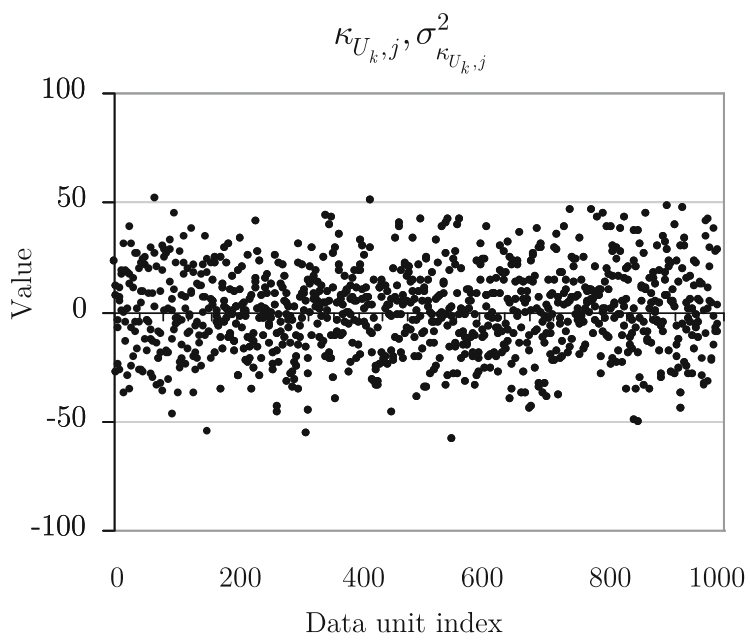

(b)

Fig. 5 a The output of GQI, $E\left(U^{-1}\left(x_{U_{k}, j}\right)\right) \in \mathcal{N}\left(0, \tilde{\sigma}_{x_{U_{k}, i}^{\prime}}^{2}\right), 0 \leq i \leq m-1, m=20$. b The $\kappa_{U_{k}, j} \in \mathcal{N}\left(0, \sigma_{\kappa U_{k}, j}^{2}\right)$ elements, $0 \leq j \leq d-1, d=1000$

In the next phase, the GQI block operates on the $x_{U_{k}, i}^{\prime}, 0 \leq i \leq m-1, m$ noisy subcarriers to determine $E\left(U^{-1}\left(x_{U_{k}, j}\right)\right)$ with minimal theoretical error.

\subsection{GQI method}

This analysis studies the distribution statistics of the GQI output and the FFT-transformed subcarrier elements.

The $E\left(U^{-1}\left(x_{U_{k}, j}\right)\right) \in \mathcal{N}\left(0, \tilde{\sigma}_{x_{U_{k}, i}^{\prime}}^{2}\right), \tilde{\sigma}_{x_{U_{k}, i}^{\prime}}^{2}=\left(F^{-1}\left(\beta_{i, \varepsilon}\right)\right)^{2} \sigma_{x_{U_{k}, i}^{\prime}}^{2}$, output of the GQI block is shown in Fig. 5a. The $i$ th subcarrier is estimated via $F^{-1}\left(x_{U_{k}, j}^{\prime}\right) * F^{-1}\left(\beta_{i, \varepsilon}\right)$.

In Fig. 5b, the $\kappa_{U_{k}, j}$ elements are depicted, $\kappa_{U_{k}, j} \in \mathcal{N}\left(0, \sigma_{\kappa_{U_{k}, j}}^{2}\right), \sigma_{\kappa_{U_{k}, j}}^{2}=\sigma_{\omega_{0}}^{2}+\sigma_{\mathcal{N}_{U_{k}, j}}^{2}$.

The GQI output elements provide vanishing magnitude error [10], which will be further utilized in the QE block, since the QE block operates on $E\left(U^{-1}\left(x_{U_{k}, j}\right)\right)$ and $\kappa_{U_{k}, j}, 0 \leq j \leq d-1$, to determine $E\left(x_{U_{k}, j}\right) \in \mathcal{N}\left(0, \tilde{\sigma}_{\omega_{0}}^{2}\right)$.

\subsection{QE method}

This analysis reveals the distribution statistics of the QE output and the expected estimation error and expected error variance.

In Fig. 6a, the output of the QE block, $E\left(x_{U_{k}, j}\right) \in \mathcal{N}\left(0, \tilde{\sigma}_{\omega_{0}}^{2}\right)$, $\tilde{\sigma}_{\omega_{0}}^{2}=\beta_{i, \varepsilon}^{2}\left(\sigma_{\omega_{0}}^{2}+\sigma_{\mathcal{N}_{U_{k}, j}}^{2}\right)$, where $\beta_{i, \varepsilon}$ is given in (70), $d=1000$. The $j$ th single carrier is estimated as $E\left(x_{U_{k, j}}\right)=\operatorname{cov}_{x_{U_{k, j}} \kappa_{U_{k}, j}} \operatorname{cov}_{\kappa_{U_{k}, j} \kappa_{U_{k}, j}}^{-1} \kappa_{U_{k, j}}$ via $E\left(U^{-1}\left(x_{U_{k}, j}\right)\right)$ and $\kappa_{U_{k}, j}$.

In Fig. 6b, the elements of the estimation error vector are depicted for $d=1000, \mathfrak{e}_{j}=x_{U_{k}, j}-E\left(x_{U_{k}, j}\right)$.

The $\operatorname{cov}_{x_{U_{k}, j} \kappa_{U_{k}, j}} \operatorname{cov}_{\kappa_{U_{k}, j} \kappa_{U_{k}, j}}^{-1} \kappa_{U_{k, j}}$ estimate approximates the $j$ th input element, $x_{U_{k}, j}$, with vanishing error, and the $\mathfrak{e}_{j}$ components converge to zero.

In Fig. 7, the $E\left(\sigma_{\xi_{j}}^{2}\right)$ expected error variances of the noise vector elements $\mathfrak{e}_{j}$ derived from $\operatorname{cov}_{\mathfrak{e} \mathfrak{e}}$ are depicted, $d=1000$. 


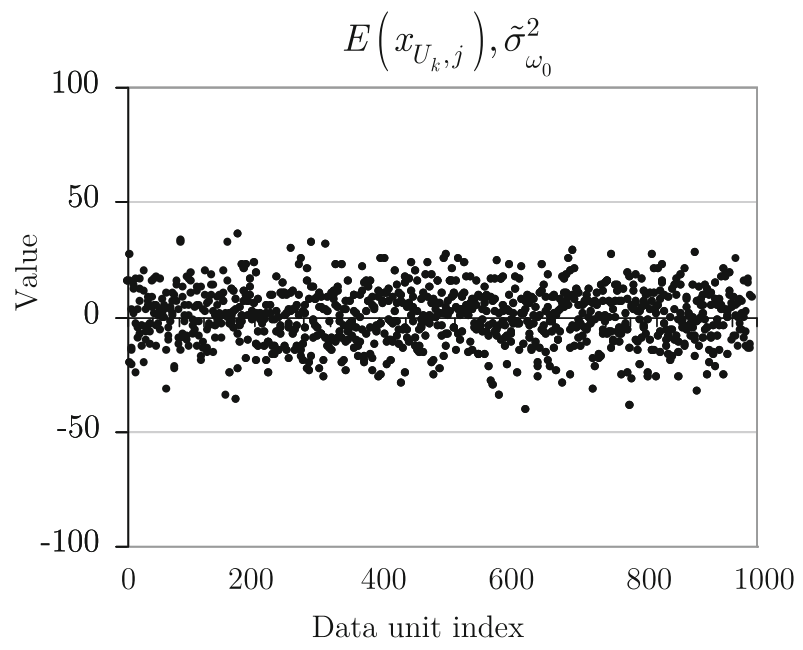

(a)

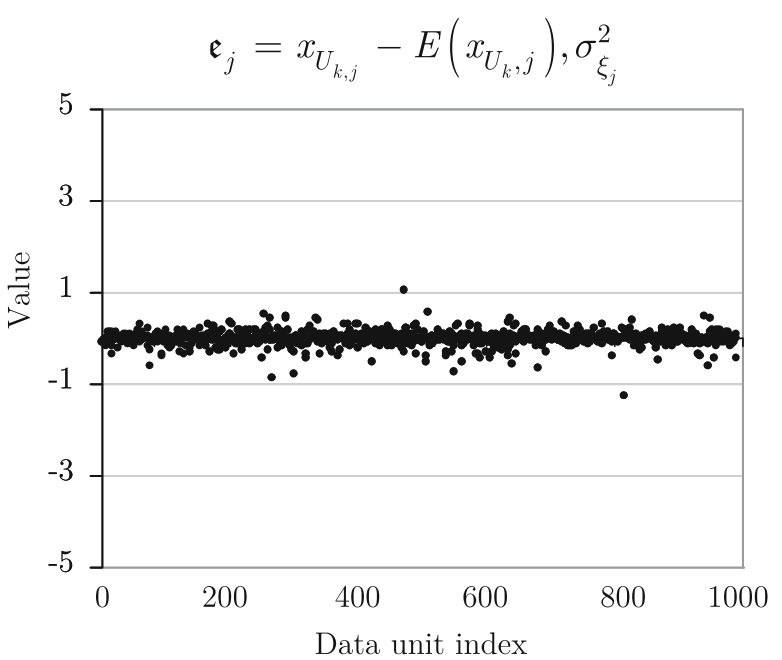

(b)

Fig. 6 a The output of QE, $E\left(x_{U_{k, j}}\right)=\operatorname{cov}_{x_{U_{k, j}} \kappa_{U_{k}, j}} \operatorname{cov}_{\kappa_{U_{k}, j} \kappa_{U_{k}, j}}^{-1} \kappa_{U_{k, j}}, 0 \leq j \leq d-1, d=1000$. b The components of error vector $\mathfrak{e}, \mathfrak{e}_{j}=x_{U_{k}, j}-E\left(x_{U_{k}, j}\right), 0 \leq j \leq d-1, d=1000$

Fig. 7 The $E\left(\sigma_{\xi_{j}}^{2}\right)$

expected error variances,

$0 \leq j \leq d-1, d=1000$

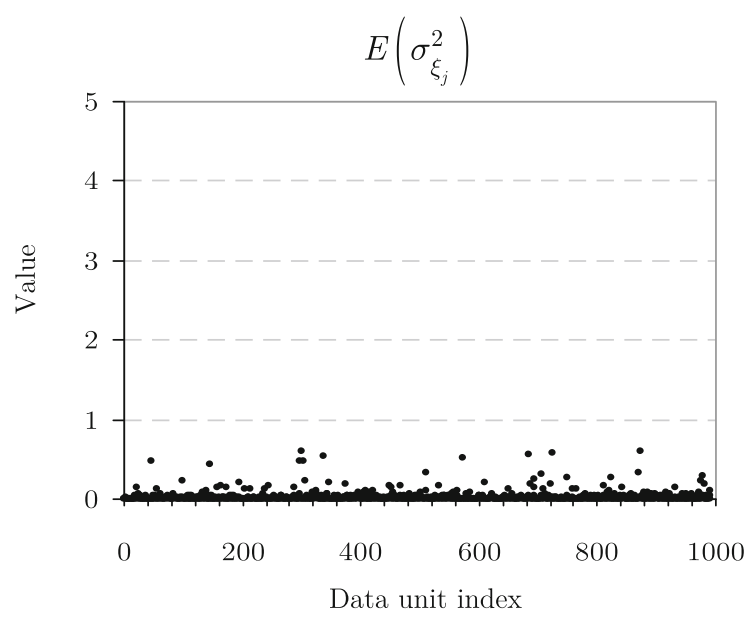

4.5 Expected error

This analysis reveals the expected error variance and errors of the QE method for a $d$-dimensional single-carrier input, in function of the number of sub-channels, $m$. For a given $m$, a $d=40$ dimensional input is considered.

In Fig. 8a, a given $\mathfrak{e}_{j}$ in function of $m$ is depicted. In Fig. 8b, the $E\left(\sigma_{\xi_{j}}^{2}\right)$ expected error variance is depicted, at $20 \leq m \leq 45, \sigma_{\mathcal{N}_{U_{k}, i}}^{2}=16, \sigma_{\omega_{0}}^{2}=225$ and $d=40$.

The QE block provides a theoretical error minimum for the quadrature estimation, based on the GQE method. As $m$ increases, $E\left(\sigma_{\xi_{j}}^{2}\right)$ converges to zero.

\section{Conclusions}

We defined the QE method for multicarrier CVQKD. The QE scheme extends the results of the GQI to evolve a minimal error estimate of the continuous input regime from noisy discrete variables and estimates. The QE model 


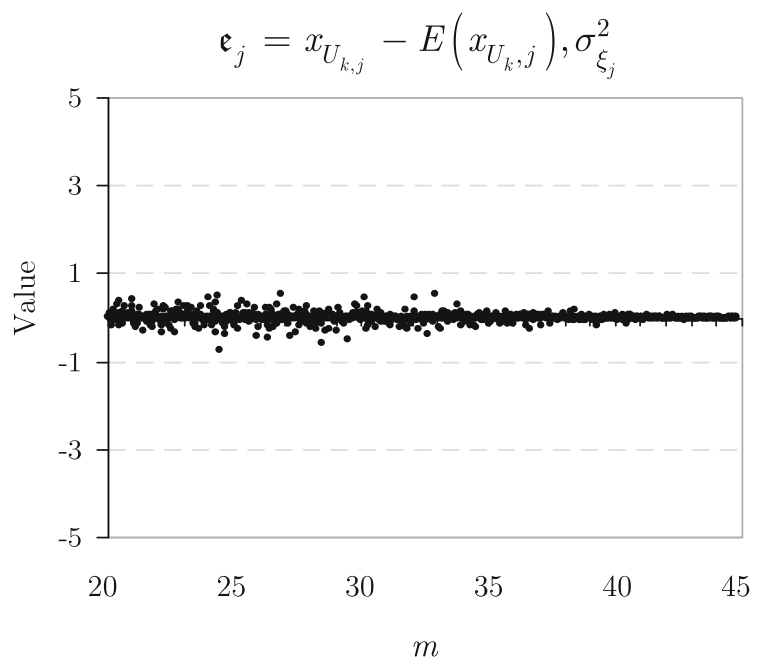

(a)

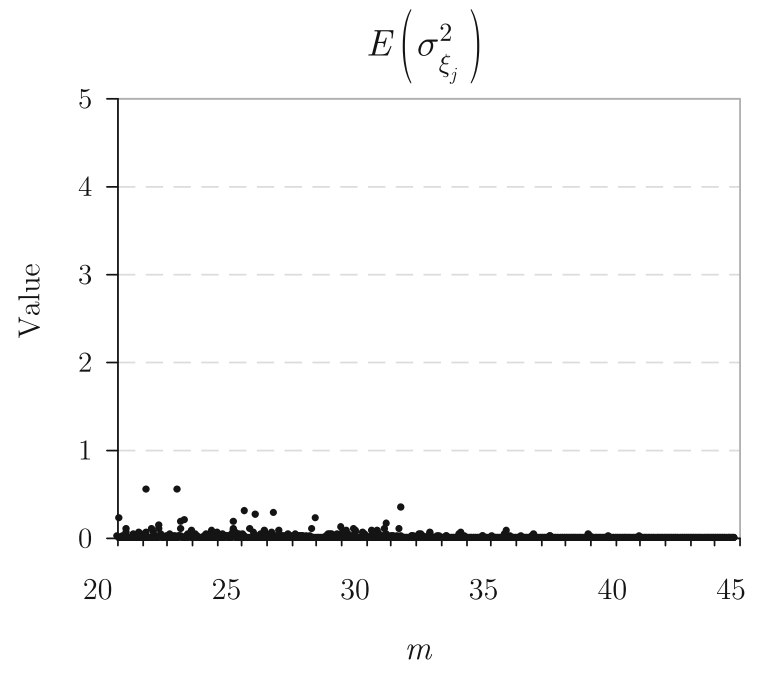

(b)

Fig. 8 a The $\mathfrak{e}_{j}$ expected error and $\mathbf{b} E\left(\sigma_{\xi_{j}}^{2}\right)$ expected error variance in function of $m, 20 \leq m \leq 45, \sigma_{\mathcal{N}_{U_{k}, i}}^{2}=16, \sigma_{\omega_{0}}^{2}=225, d=40$

achieves a theoretically minimized magnitude error to evolve the non-observable continuous variable quadratures from the measured values with a vanishing error. The QE output is always a unique and stable solution, which minimizes the overall expected estimation error for each individual component of the input. We proved that in a multicarrier CVQKD setting the QE method achieves a vanishing error as the number of subcarriers increases. We derived the corresponding expected error and expected variance via the statistical model. The QE scheme provides a viable, easily implementable, and computationally efficient way to maximize the extractable information from the observed data.

Acknowledgements Open access funding provided by Budapest University of Technology and Economics (BME). The research reported in this paper has been supported by the National Research, Development and Innovation Fund (TUDFO/51757/2019-ITM, Thematic Excellence Program). This work was partially supported by the National Research Development and Innovation Office of Hungary (Project No. 2017-1.2.1-NKP-2017-00001), by the Hungarian Scientific Research Fund - OTKA K-112125 and in part by the BME Artificial Intelligence FIKP grant of EMMI (BME FIKP-MI/SC).

Funding No relevant funding.

\section{Compliance with ethical standards}

Author contributions L.GY. designed the protocol and wrote the manuscript. L.GY. and S.I. analyzed the results. All authors reviewed the manuscript.

Conflict of interest We have no competing interests.

Ethical statement This work did not involve any active collection of human data.

Data accessibility statement This work does not have any experimental data.

Open Access This article is distributed under the terms of the Creative Commons Attribution 4.0 International License (http:// creativecommons.org/licenses/by/4.0/), which permits unrestricted use, distribution, and reproduction in any medium, provided you give appropriate credit to the original author(s) and the source, provide a link to the Creative Commons license, and indicate if changes were made. 


\section{A Appendix}

\section{A.1 Multicarrier CVQKD}

First, we summarize the basic notations of AMQD [8]. The following description assumes a single user, and the use of $n$ Gaussian sub-channels $\mathcal{N}_{i}$ for the transmission of the subcarriers, from which only $l$ sub-channels will carry valuable information.

In the single-carrier modulation scheme, the $j$ th input single-carrier state $\left|\varphi_{j}\right|=\left|x_{j}+\mathrm{i} p_{j}\right\rangle$ is a Gaussian state in the phase space $\mathcal{S}$, with i.i.d. Gaussian random position and momentum quadratures $x_{j} \in \mathcal{N}\left(0, \sigma_{\omega_{0}}^{2}\right)$, $p_{j} \in \mathcal{N}\left(0, \sigma_{\omega_{0}}^{2}\right)$, where $\sigma_{\omega_{0}}^{2}$ is the modulation variance of the quadratures (For simplicity, $\sigma_{\omega_{0}}^{2}$ is referred to as the single-carrier modulation variance, throughout). Particularly, this Gaussian single carrier is transmitted through a Gaussian quantum channel $\mathcal{N}$. In the multicarrier scenario, the information is carried by Gaussian subcarrier $\mathrm{CVs},\left|\phi_{i}\right\rangle=\left|x_{i}+\mathrm{i} p_{i}\right\rangle, x_{i} \in \mathcal{N}\left(0, \sigma_{\omega}^{2}\right), p_{i} \in \mathcal{N}\left(0, \sigma_{\omega}^{2}\right)$, where $\sigma_{\omega}^{2}$ is the modulation variance of the subcarrier quadratures, which are transmitted through a noisy Gaussian sub-channel $\mathcal{N}_{i}$. Each $\mathcal{N}_{i}$ Gaussian sub-channel is dedicated for the transmission of one Gaussian subcarrier CV from the $n$ subcarrier CVs. (Note: index $i$ refers to the subcarriers, while index $j$ to the single carriers throughout the manuscript.) The single-carrier state $\left|\varphi_{j}\right\rangle$ in the phase space $\mathcal{S}$ can be modeled as a zero-mean, circular symmetric complex Gaussian random variable $z_{j} \in \mathcal{C N}\left(0, \sigma_{\omega_{z_{j}}}^{2}\right)$, with variance $\sigma_{\omega_{z_{j}}}^{2}=\mathbb{E}\left[\left|z_{j}\right|^{2}\right]$, and with i.i.d. real and imaginary zero-mean Gaussian random components $\operatorname{Re}\left(z_{j}\right) \in \mathcal{N}\left(0, \sigma_{\omega_{0}}^{2}\right), \operatorname{Im}\left(z_{j}\right) \in \mathcal{N}\left(0, \sigma_{\omega_{0}}^{2}\right)$.

In the multicarrier CVQKD scenario, let $n$ be the number of Alice's input single-carrier Gaussian states. The $n$ input coherent states are modeled by an $n$-dimensional, zero-mean, circular symmetric complex random Gaussian vector

$\mathbf{z}=\mathbf{x}+i \mathbf{p}=\left(z_{1}, \ldots, z_{n}\right)^{T} \in \mathcal{C N}\left(0, \mathbf{K}_{\mathbf{z}}\right)$

where each $z_{j}$ can be modeled as a zero-mean, circular symmetric complex Gaussian random variable

$z_{j} \in \mathcal{C N}\left(0, \sigma_{\omega_{z_{j}}}^{2}\right), z_{j}=x_{j}+\mathrm{i} p_{j}$.

Specifically, the real and imaginary variables (i.e., the position and momentum quadratures) formulate $n$-dimensional real Gaussian random vectors, $\mathbf{x}=\left(x_{1}, \ldots, x_{n}\right)^{T}$ and $\mathbf{p}=\left(p_{1}, \ldots, p_{n}\right)^{T}$, with zero-mean Gaussian random variables with densities $f\left(x_{j}\right)$ and $f\left(p_{j}\right)$ as

$f\left(x_{j}\right)=\frac{1}{\sigma_{\omega_{0}} \sqrt{2 \pi}} \mathrm{e}^{\frac{-x_{j}^{2}}{2 \sigma_{\omega_{0}}^{2}}}, f\left(p_{j}\right)=\frac{1}{\sigma_{\omega_{0}} \sqrt{2 \pi}} \mathrm{e}^{\frac{-p_{j}^{2}}{2 \sigma_{\omega_{0}}^{2}}}$,

where $\mathbf{K}_{\mathbf{z}}$ is the $n \times n$ Hermitian covariance matrix of $\mathbf{z}$ :

$\mathbf{K}_{\mathbf{z}}=\mathbb{E}\left[\mathbf{z z}^{\dagger}\right]$,

while $\mathbf{z}^{\dagger}$ is the adjoint of $\mathbf{z}$.

For vector $\mathbf{z}$,

$\mathbb{E}[\mathbf{z}]=\mathbb{E}\left[e^{i \gamma} \mathbf{z}\right]=\mathbb{E} e^{i \gamma}[\mathbf{z}]$

holds, and

$\mathbb{E}\left[\mathbf{z z}^{T}\right]=\mathbb{E}\left[e^{i \gamma} \mathbf{z}\left(e^{i \gamma} \mathbf{z}\right)^{T}\right]=\mathbb{E} e^{i 2 \gamma}\left[\mathbf{z z}^{T}\right]$,

for any $\gamma \in[0,2 \pi]$. The density of $\mathbf{z}$ is as follows (if $\mathbf{K}_{\mathbf{z}}$ is invertible):

$f(\mathbf{z})=\frac{1}{\pi^{n} \operatorname{det} \mathbf{K}_{\mathbf{z}}} e^{-\mathbf{z}^{\dagger} \mathbf{K}_{\mathbf{z}}^{-1} \mathbf{z}}$. 
A $n$-dimensional Gaussian random vector is expressed as $\mathbf{x}=\mathbf{A s}$, where $\mathbf{A}$ is an (invertible) linear transform from $\mathbb{R}^{n}$ to $\mathbb{R}^{n}$, and $\mathbf{s}$ is an $n$-dimensional standard Gaussian random vector $\mathcal{N}(0,1)_{n}$. This vector is characterized by its covariance matrix $\mathbf{K}_{\mathbf{x}}=\mathbb{E}\left[\mathbf{x} \mathbf{x}^{T}\right]=\mathbf{A} \mathbf{A}^{T}$, and has density

$f(\mathbf{x})=\frac{1}{(\sqrt{2 \pi})^{n} \sqrt{\operatorname{det}\left(\mathbf{A A}^{T}\right)}} e^{-\frac{\mathbf{x}^{T} \mathbf{x}}{2\left(\mathbf{A A}^{T}\right)}}$.

The Fourier transformation $F(\cdot)$ of the $n$-dimensional Gaussian random vector $\mathbf{v}=\left(v_{1}, \ldots, v_{n}\right)^{T}$ results in the $n$-dimensional Gaussian random vector $\mathbf{m}=\left(m_{1}, \ldots, m_{n}\right)^{T}$, as:

$\mathbf{m}=F(\mathbf{v})=e^{\frac{-\mathbf{m}^{T} \mathbf{A} \mathbf{A}^{T} \mathbf{m}}{2}}=e^{\frac{-\sigma_{\omega_{0}}^{2}\left(m_{1}^{2}+\ldots+m_{n}^{2}\right)}{2}}$.

In the first step of AMQD, Alice applies the inverse FFT (fast Fourier transform) operation to vector $\mathbf{z}$ (see (A.1)), which results in an $n$-dimensional zero-mean, circular symmetric complex Gaussian random vector $\mathbf{d}$, $\mathbf{d} \in \mathcal{C N}\left(0, \mathbf{K}_{\mathbf{d}}\right), \mathbf{d}=\left(d_{1}, \ldots, d_{n}\right)^{T}$, as

$\mathbf{d}=F^{-1}(\mathbf{z})=e^{\frac{\mathbf{d}^{T} \mathbf{A} \mathbf{A}^{T} \mathbf{d}}{2}}=e^{\frac{\sigma_{\omega_{0}}^{2}\left(d_{1}^{2}+\ldots+d_{n}^{2}\right)}{2}}$,

where

$d_{i}=x_{d_{i}}+\mathrm{i} p_{d_{i}}, d_{i} \in \mathcal{C N}\left(0, \sigma_{d_{i}}^{2}\right)$,

where $\sigma_{\omega_{d_{i}}}^{2}=\mathbb{E}\left[\left|d_{i}\right|^{2}\right]$ and the position and momentum quadratures of $\left|\phi_{i}\right\rangle$ are i.i.d. Gaussian random variables

$\operatorname{Re}\left(d_{i}\right)=x_{d_{i}} \in \mathcal{N}\left(0, \sigma_{\omega_{i}}^{2}\right), \operatorname{Im}\left(d_{i}\right)=p_{d_{i}} \in \mathcal{N}\left(0, \sigma_{\omega_{i}}^{2}\right)$,

where $\mathbf{K}_{\mathbf{d}}=\mathbb{E}\left[\mathbf{d} \mathbf{d}^{\dagger}\right], \mathbb{E}[\mathbf{d}]=\mathbb{E}\left[e^{i \gamma} \mathbf{d}\right]=\mathbb{E} e^{i \gamma}[\mathbf{d}]$, and $\mathbb{E}\left[\mathbf{d d}^{T}\right]=\mathbb{E}\left[e^{i \gamma} \mathbf{d}\left(e^{i \gamma} \mathbf{d}\right)^{T}\right]=\mathbb{E} e^{i 2 \gamma}\left[\mathbf{d} \mathbf{d}^{T}\right]$, for any $\gamma \in[0,2 \pi]$.

The $\mathbf{T}(\mathcal{N})$ transmittance vector of $\mathcal{N}$ in the multicarrier transmission is

$\mathbf{T}(\mathcal{N})=\left[T_{1}\left(\mathcal{N}_{1}\right), \ldots, T_{n}\left(\mathcal{N}_{n}\right)\right]^{T} \in \mathcal{C}^{n}$,

where

$T_{i}\left(\mathcal{N}_{i}\right)=\operatorname{Re}\left(T_{i}\left(\mathcal{N}_{i}\right)\right)+\operatorname{iIm}\left(T_{i}\left(\mathcal{N}_{i}\right)\right) \in \mathcal{C}$,

is a complex variable, which quantifies the position and momentum quadrature transmission (i.e., gain) of the $i$ th Gaussian sub-channel $\mathcal{N}_{i}$, in the phase space $\mathcal{S}$, with real and imaginary parts

$0 \leq \operatorname{Re} T_{i}\left(\mathcal{N}_{i}\right) \leq 1 / \sqrt{2}$,

and

$0 \leq \operatorname{Im} T_{i}\left(\mathcal{N}_{i}\right) \leq 1 / \sqrt{2}$.

Particularly, the $T_{i}\left(\mathcal{N}_{i}\right)$ variable has the squared magnitude of

$\left|T_{i}\left(\mathcal{N}_{i}\right)\right|^{2}=\operatorname{Re} T_{i}\left(\mathcal{N}_{i}\right)^{2}+\operatorname{Im} T_{i}\left(\mathcal{N}_{i}\right)^{2} \in \mathbb{R}$,

where

$\operatorname{Re} T_{i}\left(\mathcal{N}_{i}\right)=\operatorname{Im} T_{i}\left(\mathcal{N}_{i}\right)$.

The Fourier-transformed transmittance of the $i$ th sub-channel $\mathcal{N}_{i}$ (resulted from CVQFT operation at Bob) is denoted by

$\left|F\left(T_{i}\left(\mathcal{N}_{i}\right)\right)\right|^{2}$. 
The $n$-dimensional zero-mean, circular symmetric complex Gaussian noise vector $\Delta \in \mathcal{C N}\left(0, \sigma_{\Delta}^{2}\right)_{n}$ of the quantum channel $\mathcal{N}$ is evaluated as

$\Delta=\left(\Delta_{1}, \ldots, \Delta_{n}\right)^{T} \in \mathcal{C} \mathbb{N}\left(0, \mathbf{K}_{\Delta}\right)$,

where

$\mathbf{K}_{\Delta}=\mathbb{E}\left[\Delta \Delta^{\dagger}\right]$.

with independent, zero-mean Gaussian random components

$\Delta_{x_{i}} \in \mathcal{N}\left(0, \sigma_{\mathcal{N}_{i}}^{2}\right)$,

and

$\Delta_{p_{i}} \in \mathcal{N}\left(0, \sigma_{\mathcal{N}_{i}}^{2}\right)$,

with variance $\sigma_{\mathcal{N}_{i}}^{2}$, for each $\Delta_{i}$ of a Gaussian sub-channel $\mathcal{N}_{i}$, which identifies the Gaussian noise of the $i$ th sub-channel $\mathcal{N}_{i}$ on the quadrature components in the phase space $\mathcal{S}$.

The CVQFT-transformed noise vector can be rewritten as

$F(\Delta)=\left(F\left(\Delta_{1}\right), \ldots, F\left(\Delta_{n}\right)\right)^{T}$,

with independent components $F\left(\Delta_{x_{i}}\right) \in \mathcal{N}\left(0, \sigma_{F\left(\mathcal{N}_{i}\right)}^{2}\right)$ and $F\left(\Delta_{p_{i}}\right) \in \mathcal{N}\left(0, \sigma_{F\left(\mathcal{N}_{i}\right)}^{2}\right)$ on the quadratures, for each $F\left(\Delta_{i}\right)$. It also defines an $n$-dimensional zero-mean, circular symmetric complex Gaussian random vector $F(\Delta) \in \mathcal{C N}\left(0, \mathbf{K}_{F(\Delta)}\right)$ with a covariance matrix

$\mathbf{K}_{F(\Delta)}=\mathbb{E}\left[F(\Delta) F(\Delta)^{\dagger}\right]$,

where $\mathbf{K}_{F(\Delta)}=\mathbf{K}_{\Delta}$, by theory. At a constant subcarrier modulation variance $\sigma_{\omega_{i}}^{2}$ for the $n$ Gaussian subcarrier $\mathrm{CVs}$, the corresponding relation is

$\frac{1}{n} \sum_{i=1}^{n} \sigma_{\omega_{i}}^{2}=\sigma_{\omega}^{2}$

where $\sigma_{\omega_{i}}^{2}$ is the modulation variance of the quadratures of the subcarrier $\left|\phi_{i}\right\rangle$ transmitted by sub-channel $\mathcal{N}_{i}$. Assuming $l$ good Gaussian sub-channels from the $n$ with constant quadrature modulation variance $\sigma_{\omega_{i}}^{2}$, where $\sigma_{\omega_{i}}^{2}=0$ for the $i$ th unused sub-channel,

$\sum_{i=1}^{l} \sigma_{\omega_{i}}^{2}=l \sigma_{\omega}^{2}<n \sigma_{\omega_{0}}^{2}$.

In particular, from the relation of (A.27), for the transmittance parameters the following relation follows at a given modulation variance $\sigma_{\omega_{0}}^{2}$, precisely,

$\left|T_{A M Q D}\left(\mathcal{N}_{i}\right)\right|^{2} \sigma_{\omega_{0}}^{2}>|T(\mathcal{N})|^{2} \sigma_{\omega_{0}}^{2}$,

where $|T(\mathcal{N})|^{2}$ is the transmittance of $\mathcal{N}$ in a single-carrier scenario, and

$$
\left|T_{A M Q D}\left(\mathcal{N}_{i}\right)\right|^{2}=\frac{1}{l} \sum_{i=1}^{l}\left|F\left(T_{i}\left(\mathcal{N}_{i}\right)\right)\right|^{2} .
$$

For the method of the determination of these $l$ Gaussian sub-channels, see [8]. Alice's $i$ th Gaussian subcarrier is expressed as

$\left|\phi_{i}\right\rangle=\left|d_{i}\right\rangle=\left|F^{-1}(z)\right\rangle$. 


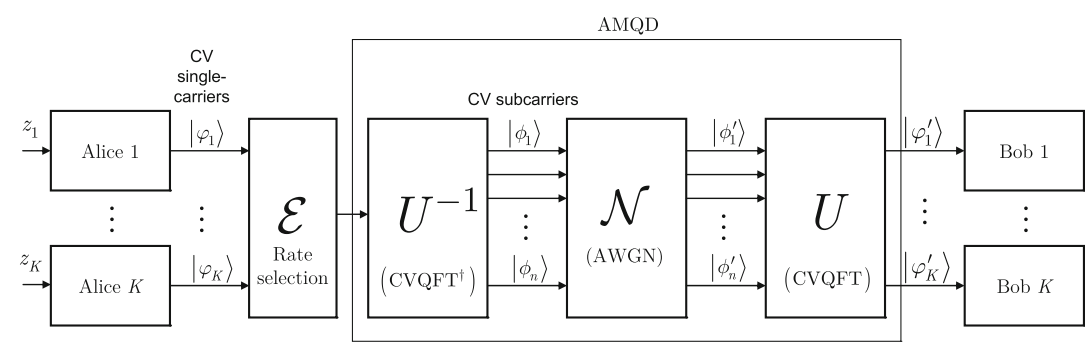

Fig. 9 The AMQD-MQA multiple access scheme with multiple independent transmitters and multiple receivers [12]. The modulated Gaussian CV single carriers are transformed by a unitary operation (inverse CVQFT) at the $\mathcal{E}$ encoder, which outputs the $n$ Gaussian subcarrier CVs for the transmission. The parties send the $\left|\varphi_{k}\right\rangle$ single-carrier Gaussian CVs with variance $\sigma_{\omega_{0, k}}^{2}$ to Alice. In the rateselection phase, the encoder determines the transmit users. The data states of the transmit users are then fed into the CVQFT $\mathrm{Cperation}^{\dagger}$ The $\left|\phi_{i}\right\rangle$ Gaussian subcarrier CVs have a variance $\sigma_{\omega}^{2}$ per quadrature components. The Gaussian CVs are decoded by the CVQFT unitary operation. Each $\left|\varphi_{k}^{\prime}\right\rangle$ is received by Bob $k$

\section{A.2 Multiuser multicarrier CVQKD}

In a MQA multiple access multicarrier CVQKD, a given user $U_{k}, k=0, \ldots, K-1$, where $K$ is the number of total users, is characterized via $m$ subcarriers, formulating an $\mathcal{M}_{U_{k}}$ logical channel of $U_{k}$,

$\mathcal{M}_{U_{k}}=\left[\mathcal{N}_{U_{k}, 0}, \ldots, \mathcal{N}_{U_{k}, m-1}\right]^{T}$,

where $\mathcal{N}_{U_{k}, i}$ is the $i$ th sub-channel of $\mathcal{M}_{U_{k}}$. For a detailed description of MQA for multicarrier CVQKD, see [12].

The general model of AMQD-MQA is depicted in Fig. 9 [12].

\section{A.3 Abbreviations}

$\begin{array}{ll}\text { AMQD } & \text { Adaptive multicarrier quadrature division } \\ \text { AWGN } & \text { Additive white Gaussian noise } \\ \text { CV } & \text { Continuous-variable } \\ \text { CVQFT } & \text { Continuous-variable quantum fourier transform } \\ \text { DGQI } & \text { Direct Gaussian quadrature inference } \\ \text { DV } & \text { Discrete-variable } \\ \text { FFT } & \text { Fast Fourier transform } \\ \text { GQI } & \text { Gaussian quadrature inference } \\ \text { ICVQFT } & \text { Inverse CVQFT } \\ \text { IFFT } & \text { Inverse fast fourier transform } \\ \text { MQA } & \text { Multiuser quadrature allocation } \\ \text { PDF } & \text { Probability density function } \\ \text { QE } & \text { Quadrature estimation } \\ \text { QFT } & \text { Quantum Fourier transform } \\ \text { QKD } & \text { Quantum key distribution } \\ \text { SNR } & \text { Signal-to-noise ratio } \\ \text { SVD } & \text { Singular value decomposition } \\ \text { WSS } & \text { Wide-sense stationary }\end{array}$

\section{A.4 Notations}

The notations of the manuscript are summarized in Table 1. 
Table 1 Summary of notations

\begin{tabular}{|c|c|}
\hline Notation & Description \\
\hline$i$ & Index for the $i$ th subcarrier Gaussian $\mathrm{CV},\left|\phi_{i}\right\rangle=x_{i}+\mathrm{i} p_{i}$ \\
\hline$j$ & Index for the $j$ th Gaussian single-carrier $\mathrm{CV},\left|\varphi_{j}\right\rangle=x_{j}+\mathrm{i} p_{j}$ \\
\hline$l$ & $\begin{array}{l}\text { Number of Gaussian sub-channels } \mathcal{N}_{i} \text { for the transmission of the Gaussian subcarriers. The overall } \\
\text { number of the sub-channels is } n \text {. The remaining } n-l \text { sub-channels do not transmit valuable information }\end{array}$ \\
\hline$x_{i}, p_{i}$ & Position and momentum quadratures of the $i$ th Gaussian subcarrier, $\left|\phi_{i}\right\rangle=x_{i}+\mathrm{i} p_{i}$ \\
\hline$x_{i}^{\prime}, p_{i}^{\prime}$ & Noisy position and momentum quadratures of Bob's $i$ th noisy subcarrier Gaussian $\mathrm{CV},\left|\phi_{i}^{\prime}\right\rangle=x_{i}^{\prime}+\mathrm{i} p_{i}^{\prime}$ \\
\hline$x_{j}, p_{j}$ & Position and momentum quadratures of the $j$ th Gaussian single-carrier $\left|\varphi_{j}\right\rangle=x_{j}+\mathrm{i} p_{j}$ \\
\hline$x_{j}^{\prime}, p_{j}^{\prime}$ & Noisy position and momentum quadratures of Bob's $j$ th recovered single-carrier Gaussian CV $\left|\varphi_{j}^{\prime}\right\rangle=x_{j}^{\prime}+\mathrm{i} p_{j}^{\prime}$ \\
\hline$x_{A, i}, p_{A, i}$ & Alice's quadratures in the transmission of the $i$ th subcarrier \\
\hline$\left|\phi_{i}\right\rangle,\left|\phi_{i}^{\prime}\right\rangle$ & Transmitted and received Gaussian subcarriers \\
\hline $\mathbf{z}$ & A $d$-dimensional input $\mathrm{CV}$ vector to transmit valuable information, $\mathbf{z} \in \mathcal{C N}\left(0, K_{\mathbf{z}}\right)$ \\
\hline $\mathbf{z}^{\prime T}$ & $\begin{array}{l}\text { A } d \text {-dimensional noisy output vector, } \mathbf{z}^{T}=\mathbf{A}^{\dagger} \mathbf{z}+\left(F^{d}(\Delta)\right)^{T}=\left(z_{0}^{\prime}, \ldots, z_{d-1}^{\prime}\right) \text {, where } \\
\quad z_{j}^{\prime}=\left(\frac{1}{l} \sum_{i=0}^{l-1} F\left(T_{j, i}\left(\mathcal{N}_{j, i}\right)\right)\right) z_{j}+F(\Delta) \in \mathcal{C N}\left(0,2\left(\sigma_{\omega_{0}}^{2}+\sigma_{\mathcal{N}}^{2}\right)\right)\end{array}$ \\
\hline$x(n)$ & A WSS (wide-sense stationary) process \\
\hline $\mathcal{A}_{x(n)}(\cdot)$ & Autocorrelation function (sequence) of $x(n)$ \\
\hline$Z$ & Number of autocorrelation coefficients \\
\hline $\mathbf{C}_{x x}$ & An $n \times n$ covariance matrix associated with $x(n)$ \\
\hline $\mathcal{P}_{x}\left(\mathrm{e}^{\mathrm{i} \omega}\right)$ & Power spectrum of $x(n)$, evaluated as $\mathcal{P}_{x}\left(\mathrm{e}^{\mathrm{i} \omega}\right)=\sum_{l=-\infty}^{+\infty} \mathcal{A}_{x(n)}(l) \mathrm{e}^{-i \omega l}$, where $\omega \in[-\pi, \pi]$ \\
\hline $\mathcal{S}_{x}\left(\mathrm{e}^{\mathrm{i} \omega}\right)$ & Spectral density of $x(n)$ \\
\hline$H(x)$ & Entropy rate of $x(n)$ \\
\hline$f_{E}(x)$ & Empirical density function \\
\hline$D_{X}(\cdot)$ & Classical relative entropy function \\
\hline$D(\cdot \| \cdot)$ & $\begin{array}{l}\text { Quantum relative entropy function, } \\
\qquad \begin{aligned} D(\rho \| \sigma) & =\operatorname{Tr}(\rho \log (\rho))-\operatorname{Tr}(\rho \log (\sigma)) \\
& =\operatorname{Tr}[\rho(\log (\rho)-\log (\sigma))]\end{aligned} \\
\text { are density matrices }\end{array}$ \\
\hline $\mathbf{S}(\rho)$ & Neumann entropy, $\mathbf{S}(\rho)=-\operatorname{Tr}(\rho \log (\rho))$ \\
\hline$N_{n}\left(f_{E}(x)\right)$ & Number of $n$-tuples $\left(x_{1}, \ldots, x_{n}\right) \in X^{n}$ with a given empirical density $f_{E}(x)$ \\
\hline $\mathfrak{L}^{p}(a, b)$ & Space of Lebesgue-measurable functions \\
\hline $\mathcal{H}_{f}(a, b)$ & Functional Hilbert space, $a=-\pi, b=\pi, \mathcal{H}_{f}(-\pi, \pi)=\mathfrak{L}^{2}(-\pi, \pi)$, with a norm $\|f\|_{2}=\frac{1}{2 \pi} \sqrt{\int_{-\pi}^{\pi}|f(x)|^{2} \mathrm{~d} x}$ \\
\hline $\mathcal{M}_{U_{k}}$ & $\begin{array}{l}\text { Logical channel of user } U_{k}, k=0, \ldots, K-1 \text {, where } K \text { is the number of total users, } \\
\mathcal{M}_{U_{k}}=\left[\mathcal{N}_{U_{k}, 0}, \ldots, \mathcal{N}_{U_{k}, m-1}\right]^{T} \text {, and } \mathcal{N}_{U_{k}, i} \text { is the } i \text { th sub-channel of } \mathcal{M}_{U_{k}}, m \text { is the number of } \\
\text { subcarriers dedicated to } U_{k}\end{array}$ \\
\hline$E\left(U^{-1}\left(\varphi_{U_{k}, j}\right)\right)$ & Estimate of $U^{-1}\left(\varphi_{U_{k}, j}\right)$ where $E(\cdot)$ stands for the estimator function, $U^{-1}$ is the inverse CVQFT operation \\
\hline$E\left(U^{-1}\left(x_{U_{k}, j}\right)\right)$ & $\begin{array}{l}\text { Estimate of } U^{-1}\left(x_{U_{k}, j}\right) \text {, where } x_{U_{k}, j} \text { is the quadrature component of } \varphi_{U_{k}, j} \text {, where } E(\cdot) \text { stands } \\
\text { for the estimator function, } U^{-1} \text { is the inverse CVQFT operation }\end{array}$ \\
\hline$M$ & Measurement operator, homodyne or heterodyne measurement \\
\hline$\theta_{\varphi_{U_{k}, j}}$ & $\begin{array}{l}\theta_{\varphi_{U_{k}, j}}=\pi / \Omega \text {, where } \Omega=\sigma_{\omega_{0}}^{2} / \sigma_{\omega}^{2}, \text { and } \sigma_{\omega_{0}}^{2}, \sigma_{\omega}^{2} \text { are the single-carrier and multicarrier modulation } \\
\quad \text { variances, } \sigma_{\omega}^{2}<\sigma_{\omega_{0}}^{2}, \Omega \geq 1, \theta_{\varphi_{U_{k}}, j} \leq \pi\end{array}$ \\
\hline
\end{tabular}


Table 1 continued

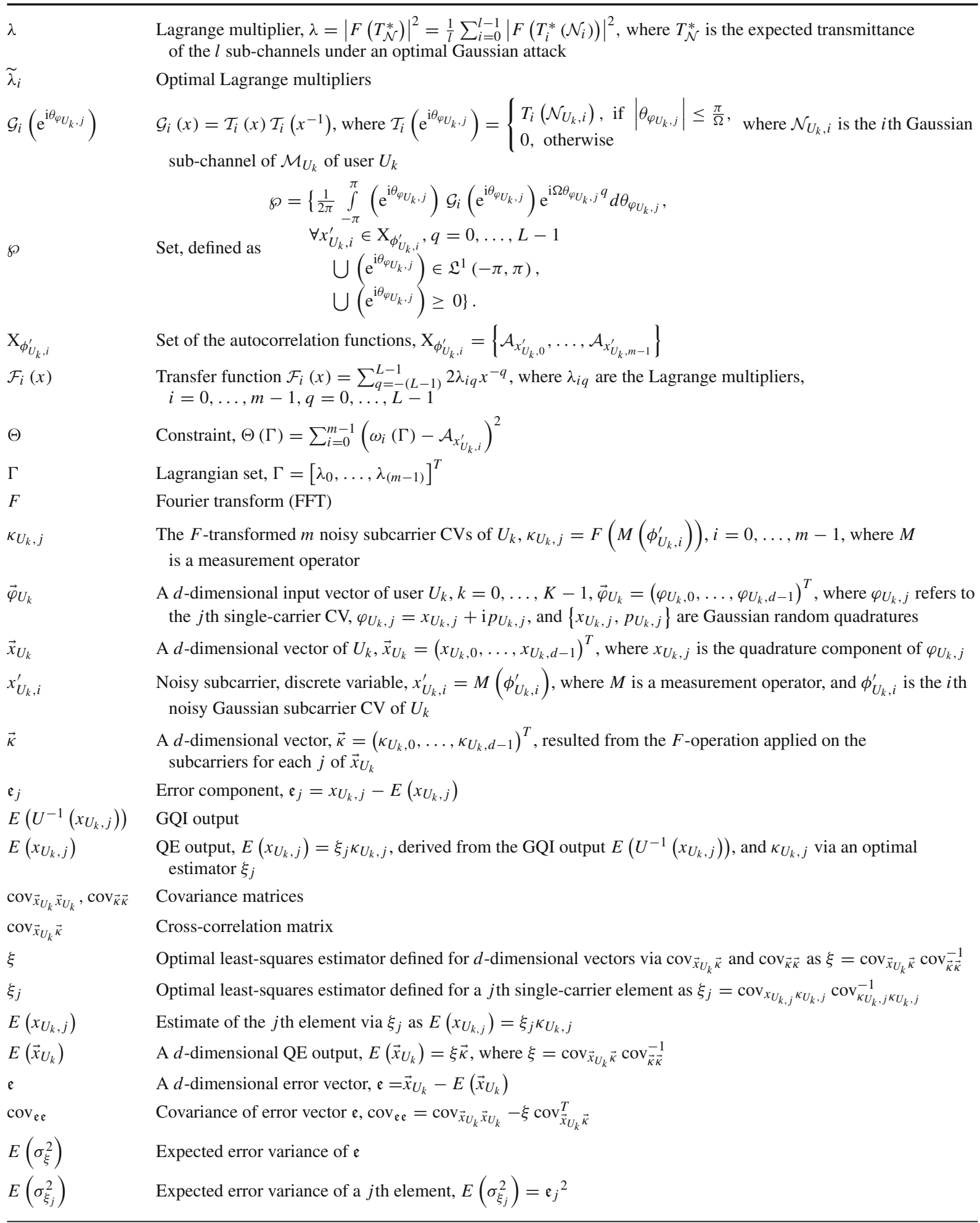


Table 1 continued

\begin{tabular}{|c|c|}
\hline$E\{\|\mathfrak{e}\|\}$ & Overall expected estimation error, $\min E\left\{\|\mathfrak{e}\|^{2}\right\}=\operatorname{Tr}\left(\operatorname{cov}_{\mathfrak{e} e}\right)$ \\
\hline$\sigma_{x_{U_{k}, i}^{\prime}}^{2}$ & Variance of $x_{U_{k}, i}^{\prime}, x_{U_{k}, i}^{\prime} \in \mathcal{N}\left(0, \sigma_{x_{U_{k}, i}^{\prime}}^{2}\right), \sigma_{x_{U_{k}, i}^{\prime}}^{2}=\sigma_{\omega}^{2}+\sigma_{\mathcal{N}_{U_{k}, i}}^{2}$ \\
\hline$E\left(U^{-1}\left(x_{U_{k}, j}\right)\right)$ & $\begin{array}{l}\text { GQI output, } E\left(U^{-1}\left(x_{U_{k}, j}\right)\right) \in \mathcal{N}\left(0, \tilde{\sigma}_{x_{U_{k}, i}^{\prime}}^{2}\right) \text {, evaluated at a direct-GQI as } \\
E\left(U^{-1}\left(x_{U_{k}, j}\right)\right)=F^{-1}\left(x_{U_{k}, j}^{\prime}\right) * F^{-1}\left(\beta_{i, \varepsilon}\right) \text {, where } * \text { is the linear convolution, while function } \\
\beta_{i, \varepsilon} \text { provides an } \varepsilon \text { minimal magnitude error } \varepsilon=\arg \min \varepsilon_{\max } \text {, where } \\
\varepsilon_{\max }=\left.\max _{\forall x}|| x_{U_{k}, j}\right|^{2}-\left|x_{U_{k}, j}^{\prime}\right|^{2} \mid \text {, and } x_{U_{k}, j}, x_{U_{k}, j}^{\prime} \text { are the input, output single-carrier } \\
\text { quadratures, } F^{-1}(\cdot) \text { is the inverse FFT operation [1], while } \beta_{i, \varepsilon}=1+\sum_{y=1}^{P} C_{y} \cos \left(y Q_{i}\right) \text {, } \\
\text { where } C_{0} \text { is arbitrarily set to unity, } P \text { is the number of } C_{y} \text { coefficients, while } \\
Q_{i}=\frac{2 \pi i}{m}, i=0, \ldots, m-1\end{array}$ \\
\hline$\sigma_{\kappa_{U_{k}, j}}^{2}$ & Variance of $\kappa_{U_{k}, j} \in \mathcal{N}\left(0, \sigma_{\kappa_{U_{k}, j}}^{2}\right), \sigma_{\kappa_{U_{k}, j}}^{2}=\sigma_{\omega_{0}}^{2}+\sigma_{\mathcal{N}_{U_{k}, j}}^{2}$ \\
\hline$\tilde{\sigma}_{\omega_{0}}^{2}$ & Variance of QE output, $E\left(x_{U_{k}, j}\right) \in \mathcal{N}\left(0, \tilde{\sigma}_{\omega_{0}}^{2}\right)$, evaluated as $\tilde{\sigma}_{\omega_{0}}^{2}=\beta_{i, \varepsilon}^{2}\left(\sigma_{\omega_{0}}^{2}+\sigma_{\mathcal{N}_{U_{k}, j}}^{2}\right)$ \\
\hline$*$ & Linear convolution operator \\
\hline $\mathfrak{f}$ & $\begin{array}{l}\text { Function, provides an } \varepsilon \text { minimal magnitude error, for } \\
\qquad E\left(U^{-1}\left(x_{U_{k}, j}\right)\right)=F^{-1}\left(x_{U_{k}, j}^{\prime}\right) * F^{-1}\left(\beta_{i}\right), i=0, \ldots, m-1\end{array}$ \\
\hline$\varepsilon$ & $\begin{array}{l}\text { Minimal magnitude error, } \varepsilon=\arg \min \varepsilon_{\max } \text {, where } \varepsilon_{\max }=\left.\max _{\forall x}|| x_{U_{k}, j}\right|^{2}-\left|x_{U_{k}, j}^{\prime}\right|^{2} \mid, x_{U_{k}, j}, \\
x_{U_{k}, j}^{\prime} \text { are the input, output single-carrier quadratures, } F^{-1}(\cdot) \text { is the inverse FFT operation }\end{array}$ \\
\hline $\mathbf{x}_{U_{k}, i}$ & Input subcarrier vector, $\mathbf{x}_{U_{k}, i}=\left(x_{U_{k}, 0}, \ldots, x_{U_{k}, m-1}\right)^{T}$ \\
\hline $\mathbf{x}_{U_{k}, i}^{\prime}$ & Output subcarrier vector, $\mathbf{x}_{U_{k}, i}^{\prime}=\left(x_{U_{k}, 0}^{\prime}, \ldots, x_{U_{k}, m-1}^{\prime}\right)^{T}$ \\
\hline $\mathrm{A}_{i}$ & $\begin{array}{l}\text { Operator, } \mathrm{A}_{i}=2 \sigma_{\omega}^{2} \frac{i}{m}, i=0, \ldots, \frac{m}{2} \text {, where } \sigma_{\omega}^{2} \text { is the subcarrier modulation } \\
\text { variance, } m \text { is the number of subcarriers of } U_{k}\end{array}$ \\
\hline$\alpha, \alpha_{\varepsilon}$ & $\alpha=m \sum_{i=0}^{m-1} \beta_{i}^{2}, \alpha_{\varepsilon}=m \sum_{z=0}^{m-1} \beta_{z, \varepsilon}^{2}$ \\
\hline$\beta_{i}$ & $\begin{array}{l}\text { Parameter, defined to evaluate function } \mathfrak{f}(s) \text { as } \\
\mathfrak{f}(s)=\frac{1}{\alpha}\left|\sum_{i=0}^{m-1} \beta_{i} \mathrm{e}^{\frac{\mathrm{i} 2 \pi i s}{m}}\right|^{2}=\frac{1}{\alpha}\left|\int_{-m / 2}^{m / 2} \cos \left(\frac{2 \pi s i}{m}\right) \beta\left(i-\frac{m}{2}\right) d i\right|^{2}\end{array}$ \\
\hline$\beta_{i, \varepsilon}$ & $\begin{array}{l}\text { Function to minimize } \varepsilon, \beta_{i, \varepsilon}=1+\sum_{y=1}^{P} C_{y} \cos \left(y Q_{i}\right) \text {, where } C_{0} \text { is arbitrarily set to } \\
\text { unity, } P \text { is the number of } C_{y} \text { coefficients, while } Q_{i}=\frac{2 \pi i}{m}, i=0, \ldots, m-1\end{array}$ \\
\hline$P\left(\mathcal{M}_{U_{k}}\right)$ & Statistical private classical information of $U_{k}$ \\
\hline$S\left(\mathcal{M}_{U_{k}}\right)$ & Statistical secret key rate of $U_{k}$ \\
\hline$\hat{x}_{(j: Z), U_{k}}, \hat{x}_{(j: Z), U_{k}}^{\prime}, \hat{x}_{(j: Z), E}^{\prime}$ & Optimal quadratures of Alice, Bob and Eve, obtained at $Z$ autocorrelation coefficients \\
\hline $\mathcal{X}_{\mathrm{AB}}$ & Holevo quantity of Bob's output \\
\hline $\mathcal{X}_{\mathrm{BE}}$ & Holevo information leaked to the Eve in a reverse reconciliation \\
\hline$\rho_{k}^{\mathrm{AB}}$ & Bob's optimal output density matrix \\
\hline$\rho_{k}^{\mathrm{BE}}$ & Eve's optimal density matrix, at a reverse reconciliation \\
\hline$\sigma^{\mathrm{AB}}$ & Bob's optimal output average density matrix \\
\hline$\sigma^{\mathrm{BE}}$ & Eve's average density matrix, at a reverse reconciliation \\
\hline$U_{K_{\text {out }}}$ & $\begin{array}{l}\text { The unitary CVQFT operation, } U_{K_{\text {out }}}=\frac{1}{\sqrt{K_{\text {out }}}} \mathrm{e}^{\frac{-\mathrm{i} 2 \pi i k}{K_{\text {out }}}} \\
i, k=0, \ldots, K_{\text {out }}-1, K_{\text {out }} \times K_{\text {out }} \text { unitary matrix }\end{array}$ \\
\hline$U_{K_{\text {in }}}$ & $\begin{array}{l}\text { The unitary inverse CVQFT operation, } U_{K_{\text {in }}}=\frac{1}{\sqrt{K_{\text {in }}}} \mathrm{e}^{\frac{\mathrm{i} 2 \pi i k}{K_{\text {in }}}} \\
i, k=0, \ldots, K_{\text {in }}-1, K_{\text {in }} \times K_{\text {in }} \text { unitary matrix }\end{array}$ \\
\hline
\end{tabular}


Table 1 continued

$z \quad$ The variable of a single-carrier Gaussian CV state, $z \in \mathcal{C N}\left(0, \sigma_{z}^{2}\right),\left|\varphi_{i}\right\rangle \in \mathcal{S}$. Zero-mean, circular symmetric complex Gaussian random variable, $\sigma_{z}^{2}=\mathbb{E}\left[|z|^{2}\right]=2 \sigma_{\omega_{0}}^{2}$, with i.i.d. zero mean, Gaussian random quadrature components $x, p \in \mathcal{N}\left(0, \sigma_{\omega_{0}}^{2}\right)$, where $\sigma_{\omega_{0}}^{2}$ is the variance

$\Delta$

d

$F^{-1}(\cdot)$

$F(\cdot)$

$F^{-1}(\cdot)$

$\sigma_{\omega_{0}}^{2}$

$\sigma_{\omega}^{2}$

$\left|\phi_{i}\right\rangle$

$\left|\varphi_{k, i}\right\rangle$

$\mathcal{N}$

$\mathcal{N}_{i}$

$T(\mathcal{N})$

$T_{i}\left(\mathcal{N}_{i}\right)$

$T_{\text {Eve }}$

$T_{\text {Eve, } i}$

Z

d

$\mathbf{y}_{k}$

$y_{k, m}$

$F(\mathbf{T}(\mathcal{N}))$
The noise variable of the Gaussian channel $\mathcal{N}, \Delta \in \mathcal{C N}\left(0, \sigma_{\Delta}^{2}\right)$, with i.i.d. zero-mean, Gaussian random noise components on the position and momentum quadratures $\Delta_{x}, \Delta_{p} \in \mathcal{N}\left(0, \sigma_{\mathcal{N}}^{2}\right)$, $\sigma_{\Delta}^{2}=\mathbb{E}\left[|\Delta|^{2}\right]=2 \sigma_{\mathcal{N}}^{2}$

The variable of a Gaussian subcarrier CV state, $d \in \mathcal{C N}\left(0, \sigma_{d}^{2}\right)$,

$\left|\phi_{i}\right\rangle \in \mathcal{S}$. Zero-mean, circular symmetric Gaussian random variable, $\sigma_{d}^{2}=\mathbb{E}\left[|d|^{2}\right]=2 \sigma_{\omega}^{2}$, with i.i.d. zero mean, Gaussian random quadrature components $x_{d}, p_{d} \in \mathcal{N}\left(0, \sigma_{\omega}^{2}\right)$, where $\sigma_{\omega}^{2}$ is the (constant) modulation variance of the Gaussian subcarrier CV state

The inverse CVQFT transformation, $F^{-1}(\cdot)=\operatorname{CVQFT}^{\dagger}(\cdot)$, applied by the encoder, continuous-variable unitary operation

The CVQFT transformation, $F(\cdot)=\operatorname{CVQFT}(\cdot)$, applied by the decoder, continuous-variable unitary operation

Inverse FFT transform, $F^{-1}(\cdot)=\operatorname{IFFT}(\cdot)$, applied by the encoder

Single-carrier modulation variance

Multicarrier modulation variance, $\sigma_{\omega}^{2}=\frac{1}{l} \sum_{l} \sigma_{\omega_{i}}^{2}$. Average modulation variance of the $l$ Gaussian sub-channels $\mathcal{N}_{i}$

The $i$ th Gaussian subcarrier CV of user $U_{k}$, where IFFT stands for the Inverse Fast Fourier Transform,

$\left|\phi_{i}\right\rangle \in \mathcal{S}, \begin{aligned}\left|\phi_{i}\right\rangle & =\mid \begin{array}{l}\left.\operatorname{IFFT}\left(z_{k, i}\right)\right\rangle \\ \left.F^{-1}\left(z_{k, i}\right)\right\rangle=\left|d_{i}\right\rangle,\end{array}, d_{i} \in \mathcal{C N}\left(0, \sigma_{d_{i}}^{2}\right), \sigma_{d_{i}}^{2}=\mathbb{E}\left[\left|d_{i}\right|^{2}\right], d_{i}=x_{d_{i}}+\mathrm{i} p_{d_{i}},\end{aligned}$

$x_{d_{i}} \in \mathcal{N}\left(0, \sigma_{\omega_{F}}^{2}\right), p_{d_{i}} \in \mathcal{N}\left(0, \sigma_{\omega_{F}}^{2}\right)$ are i.i.d. zero-mean Gaussian random quadrature components, and $\sigma_{\omega_{F}}^{2}$ is the variance of the Fourier transformed Gaussian state

The decoded single-carrier CV of user $U_{k}$ from the subcarrier CV, $\left|\varphi_{k, i}\right\rangle=\mathrm{CVQFT}\left(\left|\phi_{i}\right\rangle\right)$, expressed as $F\left(\left|d_{i}\right\rangle\right)=\left|F\left(F^{-1}\left(z_{k, i}\right)\right)\right\rangle=\left|z_{k, i}\right\rangle$

Gaussian quantum channel

An $i$ th Gaussian sub-channels, $i=0, \ldots, n-1$

Channel transmittance, normalized complex random variable, $T(\mathcal{N})=\operatorname{Re} T(\mathcal{N})+\operatorname{iIm} T(\mathcal{N}) \in \mathcal{C}$. The real part identifies the position quadrature transmission, the imaginary part identifies the transmittance of the position quadrature

Transmittance coefficient of Gaussian sub-channel $\mathcal{N}_{i}, T_{i}\left(\mathcal{N}_{i}\right)=\operatorname{Re}\left(T_{i}\left(\mathcal{N}_{i}\right)\right)+\operatorname{iIm}\left(T_{i}\left(\mathcal{N}_{i}\right)\right) \in \mathcal{C}$, quantifies the position and momentum quadrature transmission, with (normalized) real and imaginary parts $0 \leq \operatorname{Re} T_{i}\left(\mathcal{N}_{i}\right) \leq 1 / \sqrt{2}, 0 \leq \operatorname{Im} T_{i}\left(\mathcal{N}_{i}\right) \leq 1 / \sqrt{2}$, where $\operatorname{Re} T_{i}\left(\mathcal{N}_{i}\right)=\operatorname{Im} T_{i}\left(\mathcal{N}_{i}\right)$

Eve's transmittance, $T_{E v e}=1-T(\mathcal{N})$

Eve's transmittance for the $i$ th subcarrier $\mathrm{CV}$

A $d$-dimensional, zero-mean, circular symmetric complex random Gaussian vector that models $d$ Gaussian CV input states, $\mathbf{z}=\mathbf{x}+\mathrm{ip}=\left(z_{0}, \ldots, z_{d-1}\right)^{T}, \mathcal{C N}\left(0, \mathbf{K}_{\mathbf{z}}\right), \mathbf{K}_{\mathbf{z}}=\mathbb{E}\left[\mathbf{z z}^{\dagger}\right]$, where $z_{j}=x_{j}+\mathrm{i} p_{j}, \mathbf{x}=\left(x_{0}, \ldots, x_{d-1}\right)^{T}$, $\mathbf{p}=\left(p_{0}, \ldots, p_{d-1}\right)^{T}, x_{j} \in \mathcal{N}\left(0, \sigma_{\omega_{0}}^{2}\right), p_{j} \in \mathcal{N}\left(0, \sigma_{\omega_{0}}^{2}\right)$ i.i.d. zero-mean Gaussian random variables

An $l$-dimensional, zero-mean, circular symmetric complex random Gaussian vector, $\mathbf{d}=F^{-1}(z), \mathcal{C N}\left(0, \mathbf{K}_{\mathbf{d}}\right)$, $\mathbf{K}_{\mathbf{d}}=\mathbb{E}\left[\mathbf{d d}^{\dagger}\right], \mathbf{d}=\left(d_{0}, \ldots, d_{l-1}\right)^{T}, d_{i}=x_{i}+\mathrm{i} p_{i}, x_{i}, p_{i} \in \mathcal{N}\left(0, \sigma_{\omega_{F}}^{2}\right)$ are i.i.d. zero-mean Gaussian random variables. The $i$ th component is $d_{i} \in \mathcal{C N}\left(0, \sigma_{d_{i}}^{2}\right), \sigma_{d_{i}}^{2}=\mathbb{E}\left[\left|d_{i}\right|^{2}\right]$

A $d$-dimensional zero-mean, circular symmetric complex Gaussian random vector, $\mathbf{y}_{k} \in \mathcal{C N}\left(0, \mathbb{E}\left[\mathbf{y}_{k} \mathbf{y}_{k}^{\dagger}\right]\right)$

The $m$ th element of the $k$ th user's vector $y_{k}$, expressed as $y_{k, m}=\sum_{l} F\left(T_{i}\left(\mathcal{N}_{i}\right)\right) F\left(d_{i}\right)+F\left(\Delta_{i}\right)$

Fourier transform of $\mathbf{T}(\mathcal{N})=\left[T_{0}\left(\mathcal{N}_{0}\right) \ldots, T_{l-1}\left(\mathcal{N}_{l-1}\right)\right]^{T} \in \mathcal{C}^{l}$, the complex transmittance vector 
Table 1 continued

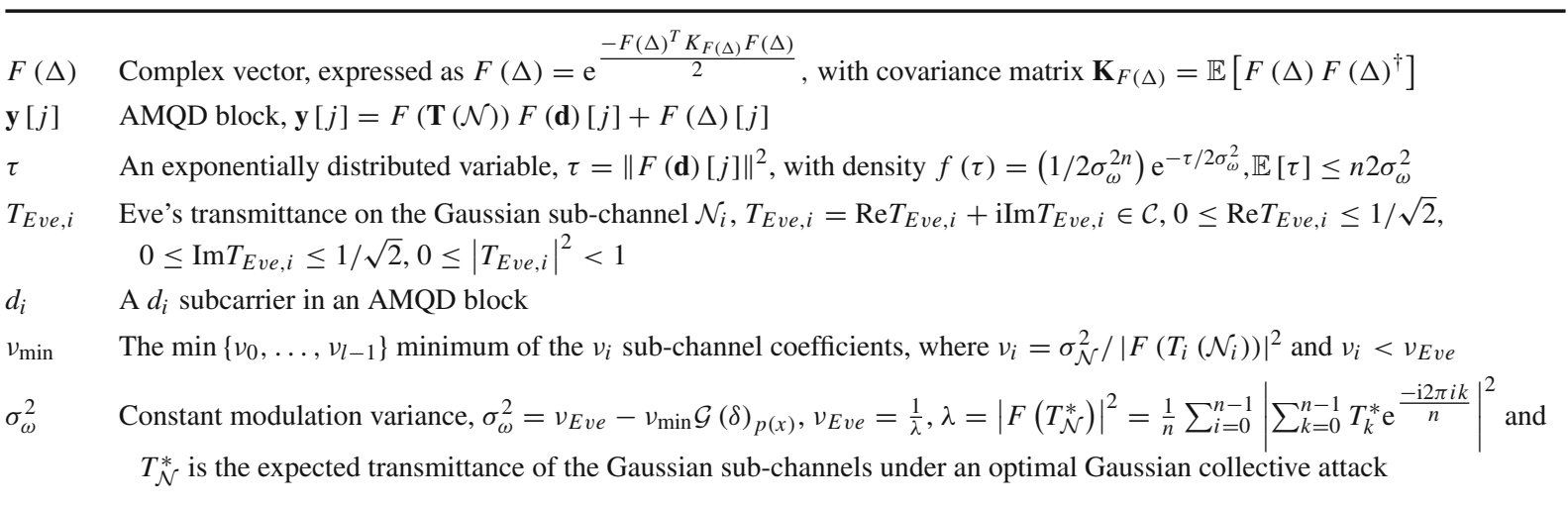

\section{References}

1. Adcock, M.R.A., Hoyer, P., Sanders, B.C.: Limitations on continuous-variable quantum algorithms with Fourier transforms. N. J. Phys. 11, 103035 (2009)

2. Bacsardi, L.: On the way to quantum-based satellite communication. IEEE Commun. Mag. 51(08), 50-55 (2013)

3. Biamonte, J., et al.: Quantum machine learning. Nature 549, 195-202 (2017)

4. Garcia-Patron, R., Cerf, N.J.: Unconditional optimality of Gaussian attacks against continuous-variable quantum key distribution. Phys. Rev. Lett. 97, 190503 (2006)

5. Grosshans, F.: Collective attacks and unconditional security in continuous variable quantum key distribution. Phys. Rev. Lett. 94, 20504 (2005)

6. Grosshans, F., Cerf, N.J., Wenger, J., Tualle-Brouri, R., Grangier, P.: Virtual entanglement and reconciliation protocols for quantum cryptography with continuous variables. Quant. Inf. Comp. 3, 535-552 (2003)

7. Gyongyosi, L.: Singular value decomposition assisted multicarrier continuous-variable quantum key distribution. Theor. Comput. Sci. 1, 1-29 (2019). https://doi.org/10.1016/j.tcs.2019.07.029

8. Gyongyosi, L., Imre, S.: Adaptive multicarrier quadrature division modulation for long-distance continuous-variable quantum key distribution. In: Proc. SPIE 9123 quantum information and computation XII, “912307”. From conference volume 9123 quantum information and computation XII, Baltimore (2014) https://doi.org/10.1117/12.2050095

9. Gyongyosi, L., Imre, S.: Diversity space of multicarrier continuous-variable quantum key distribution. Int. J. Commun. Syst. (Wiley) (2019). https://doi.org/10.1002/dac.4003

10. Gyongyosi, L., Imre, S.: Gaussian quadrature inference for multicarrier continuous-variable quantum key distribution. In: Quantum studies: mathematics and foundations, pp. 1-34. Springer (2019). https://doi.org/10.1007/s40509-019-00183-9

11. Gyongyosi, L., Imre, S.: Geometrical analysis of physically allowed quantum cloning transformations for quantum cryptography, information sciences, pp. 1-23. Elsevier (2014) https://doi.org/10.1016/j.ins.2014.07.010

12. Gyongyosi, L., Imre, S.: Multiple access multicarrier continuous-variable quantum key distribution, chaos, solitons and fractals. Elsevier (2018) https://doi.org/10.1016/j.chaos.2018.07.006, ISSN: 0960-0779

13. Gyongyosi, L., Imre, S.: Secret key rate proof of multicarrier continuous-variable quantum key distribution. Int. J. Commun. Syst. (Wiley) (2018). https://doi.org/10.1002/dac.3865

14. Gyongyosi, L., Imre, S.L: Proceedings volume 8997 advances in photonics of quantum computing, memory, and communication VII. (2014). https://doi.org/10.1117/12.2038532

15. Gyongyosi, L., Imre, S.: Long-distance continuous-variable quantum key distribution with advanced reconciliation of a Gaussian modulation. In: Proceedings Volume 8997, Advances in Photonics of Quantum Computing, Memory, and Communication VII; 89970C. (2014). https://doi.org/10.1117/12.2038532

16. Gyongyosi, L., Imre, S.: Low-dimensional reconciliation for continuous-variable quantum key distribution. Appl. Sci. (2018) https://doi.org/10.3390/app8010087 ISSN 2076-3417

17. Gyongyosi, L.: Diversity extraction for multicarrier continuous-variable quantum key distribution. In: Proceedings of the 2016 24th European signal processing conference (EUSIPCO 2016), (2016)

18. Gyongyosi, L., Imre, S.: Eigenchannel decomposition for continuous-variable quantum key distribution. In: Proceedings Volume 9377, Advances in Photonics of Quantum Computing, Memory, and Communication VIII; 937711. (2015). https://doi.org/10.1117/ 12.2076532

19. Gyongyosi, L., Imre, S.: Singular layer transmission for continuous-variable quantum key distribution. In: IEEE Photonics Conference (IPC) 2014. IEEE (2014). https://doi.org/10.1109/IPCon.2014.6995246 
20. Gyongyosi, L., Imre, S.: Adaptive Gaussian quadrature detection for continuous-variable quantum key distribution. In: Proceedings Volume 9762, Advances in Photonics of Quantum Computing, Memory, and Communication IX; 97620E. (2016). https://doi.org/ $10.1117 / 12.2211743$

21. Gyongyosi, L., Imre, S.: Gaussian quadrature inference for multicarrier continuous-variable quantum key distribution, vol. XIV. SPIE Quantum Information and Computation, Baltimore (2016). (17-21 Apr 2016 )

22. Gyongyosi, L., Imre, S.: Decentralized Base-Graph Routing for the Quantum Internet. Phys. Rev. A. Am. Phys. Soc. (2018). https:// doi.org/10.1103/PhysRevA.98.022310

23. Gyongyosi, L., Imre, S.: Entanglement-gradient routing for quantum networks. Sci. Rep. Nat. (2017) https://doi.org/10.1038/ s41598-017-14394-w

24. Gyongyosi, L., Imre, S.: Entanglement availability differentiation service for the quantum internet. Sci. Rep. Nat. (2018)https:// doi.org/10.1038/s41598-018-28801-3

25. Gyongyosi, L., Imre, S.: Secret key rates of free-space optical continuous-variable quantum key distribution. Int. J. Commun. Syst. (2019). https://doi.org/10.1002/dac.4152

26. Gyongyosi, L., Imre, S., Nguyen, H.V.: A survey on quantum channel capacities. IEEE Commun. Surv. Tutor. 99, 1 (2018). https:// doi.org/10.1109/COMST.2017.2786748

27. Heinzel, G., Rudiger, A., Schilling, R.: Spectrum and spectral density estimation by the Discrete Fourier transform (DFT), including a comprehensive list of window functions and some new at-top windows, (2002). http://hdl.handle.net/11858/ 00-001M-0000-0013-557A-5

28. Imre, S., Gyongyosi, L.: Advanced quantum communications-an engineering approach. Wiley-IEEE Press, New Jersey (2012)

29. Imre, S., Balazs, F.: Quantum computing and communications-an engineering approach. Wiley (2005). ISBN 0-470-86902-X

30. Jahromi, O.S.: Multirate statistical signal processing. Springer, Dordrecht (2007). ISBN-10 1-4020-5316-9

31. Jouguet, P., Kunz-Jacques, S., Leverrier, A., Grangier, P., Diamanti, E.: Experimental demonstration of long-distance continuousvariable quantum key distribution, (2012) arXiv:1210.6216v1

32. Kay, S.: Fundamentals of statistical signal processing, Volumes I-III. Prentice Hall (2013). ISBN-13: 978-0133457117, ISBN10:133457117

33. Kiktenko, E.O., Pozhar, N.O., Anufriev, M.N., Trushechkin, A.S., Yunusov, R.R., Kurochkin, Y.V., Lvovsky, A.I., Fedorov, A.K.: Quantum-secured blockchain. Quant. Sci. Technol. 3, 35004 (2018)

34. Kimble, H.J.: The quantum Internet. Nature 453, 1023-1030 (2008). https://doi.org/10.1038/nature07127

35. Laudenbach, F., Pacher, C., Fred Fung, C.-H., Poppe, A., Peev, M., Schrenk, B., Hentschel, M., Walther, P., Hubel, H.: Continuousvariable quantum key distribution with Gaussian modulation-the theory of practical implementations. Adv. Quant. Technol. 1(1), 1-37 (2018). https://doi.org/10.1002/qute.201800011

36. Laurenza, R., Pirandola, S.: General bounds for sender-receiver capacities in multipoint quantum communications. Phys. Rev. A 96, 32318 (2017)

37. Lloyd, S.: Capacity of the noisy quantum channel. Phys. Rev. A 55, 1613-1622 (1997)

38. Lloyd, S., Mohseni, M., Rebentrost, P.: Quantum algorithms for supervised and unsupervised machine learning (2013). arXiv: 1307.0411

39. Lloyd, S., Mohseni, M., Rebentrost, P.: Quantum principal component analysis. Nat. Phys. 10, 631 (2014)

40. Lloyd, S., Shapiro, J.H., Wong, F.N.C., Kumar, P., Shahriar, S.M., Yuen, H.P.: Infrastructure for the quantum internet. ACM SIGCOMM Comput. Commun. Rev. 34, 9-20 (2004)

41. Middlet, D.: An introduction to statistical communication theory: an IEEE press Classic Reissue, Hardcover. IEEE (1960) ISBN-10: 780311787 ISBN-13: 978-0780311787

42. Muralidharan, S., Kim, J., Lutkenhaus, N., Lukin, M.D., Jiang, L.: Ultrafast and fault-tolerant quantum communication across long distances. Phys. Rev. Lett. 112, 250501 (2014)

43. Navascues, M., Acin, A.: Security bounds for continuous variables quantum key distribution. Phys. Rev. Lett. 94, 20505 (2005)

44. Navascues, M., Grosshans, F., Acin, A.: Optimality of Gaussian attacks in continuous-variable quantum cryptography. Phys. Rev. Lett. 97, 190502 (2006)

45. Petz, D.: Quantum information theory and quantum statistics. Springer, Heidelberg (2008). Hiv: 6

46. Pirandola, S.: Capacities of repeater-assisted quantum communications, (2016) arXiv:1601.00966

47. Pirandola, S.: End-to-end capacities of a quantum communication network. Commun. Phys. 2, 51 (2019)

48. Pirandola, S., Braunstein, S.L., Laurenza, R., Ottaviani, C., Cope, T.P.W., Spedalieri, G., Banchi, L.: Theory of channel simulation and bounds for private communication. Quant. Sci. Technol. 3, 35009 (2018). https://doi.org/10.1088/2058-9565/aac394

49. Pirandola, S., Braunstein, S.L., Lloyd, S.: Characterization of collective Gaussian attacks and security of coherent-state quantum cryptography. Phys. Rev. Lett. 101, 200504 (2008)

50. Pirandola, S., Garcia-Patron, R., Braunstein, S.L., Lloyd, S.: Direct and reverse secret-key capacities of a quantum channel. Phys. Rev. Lett. 102, 50503 (2009)

51. Pirandola, S., Mancini, S., Lloyd, S., Braunstein, S.L.: Continuous-variable quantum cryptography using two-way quantum communication. Nat. Phys. 4, 726-730 (2008)

52. Pirandola, S., Laurenza, R., Ottaviani, C., Banchi, L.: Fundamental limits of repeaterless quantum communications. Nat. Commun. 8, 15043 (2017). https://doi.org/10.1038/ncomms 15043

53. Pirandola, S., Serafini, A., Lloyd, S.: Correlation matrices of two-mode bosonic systems. Phys. Rev. A 79, 52327 (2009) 
54. Press, W. H., Teukolsky, S. A., Vetterling, W. T., Flannery, B. P.: Numerical recipes in C: the art of scientific computing. Cambridge University Press (1993). ISBN: 0-521-43108-5

55. Shieh, W., Djordjevic, I.: OFDM for optical communications. Elsevier (2010). Hardcover ISBN: 9780123748799, eBook ISBN: 9780080952062

56. Shor, P.W.: Scheme for reducing decoherence in quantum computer memory. Phys. Rev. A 52, R2493-R2496 (1995)

57. Tse, D., Viswanath, P.: Fundamentals of wireless communication. Cambridge University Press (2005). ISBN-13: 978-0521845274, ISBN-10: 521845270

58. Van Meter, R.: Quantum Networking. Wiley (2014). ISBN-13: 9781848215375 , ISBN-10: 9781848215375

59. Weedbrook, C., Pirandola, S., Lloyd, S., Ralph, T.: Quantum cryptography approaching the classical limit. Phys. Rev. Lett. 105, 110501 (2010)

60. Weedbrook, C., Pirandola, S., Garcia-Patron, R., Cerf, N.J., Ralph, T., Shapiro, J., Lloyd, S.: Gaussian quantum information. Rev. Mod. Phys. 84, 621 (2012)

61. Zhang, H., Mao, Y., Huang, D., Li, J., Zhang, L., Guo, Y.: Security analysis of orthogonal-frequency-division-multiplexing-based continuous-variable quantum key distribution with imperfect modulation. Phys. Rev. A 97, 52328 (2018)

62. Zhao, W., Liao, Q., Huang, D. et al.: Performance analysis of the satellite-to-ground continuous-variable quantum key distribution with orthogonal frequency division multiplexed modulation. Quant. Inf. Proc. 18:39 (2019). https://doi.org/10.1007/ s11128-018-2147-8

Publisher's Note Springer Nature remains neutral with regard to jurisdictional claims in published maps and institutional affiliations. 